\title{
Intriguing Radical-Radical Interactions in Doubly Reduced Dimers: Cytosine Anion Radical versus Hydrogenated Cytosine Radical
}

\author{
Jing Zhao ${ }^{\text {a }}$, Ruofei Hu ${ }^{* *}$, a , Mei Wang ${ }^{\mathrm{c}}$, and Yuxiang $\mathrm{Bu}^{\text {*,b }}$
}

a. College of Life Science, Dezhou University, Dezhou 253023, People’s Republic of China.

b.School of Chemistry and Chemical Engineering, Shandong University, Jinan, 250100,

People's Republic of China

c. Department of Physics, Qufu Normal University, Qufu 273165, PR China

Supporting Information

* The correspondence should be addressed: Prof. Dr. Yuxiang Bu, E-mail: byx@sdu.edu.cn

** Dr. Ruofei Hu, E-mail: huruofei@tju.edu.cn 
Table S1. Binding energies $(\mathrm{kcal} / \mathrm{mol})$ of $\mathrm{C}^{\circ-} \mathrm{C}^{\circ-}$ series, $\mathrm{CH} 5^{\circ} \mathrm{CH} 5^{\circ}$ series, $\mathrm{CH} 6{ }^{\circ} \mathrm{CH} 6^{\circ}$ series, $\mathrm{CH}^{\circ} \mathrm{CH} 5^{\circ}$ series, $\mathrm{CH} 5^{\circ} \mathrm{C}^{\circ-}$ series, and $\mathrm{CH} 6^{\circ} \mathrm{C}^{\circ-}$ series obtained at the $\mathrm{B} 3 \mathrm{LYP} / 6-311+\mathrm{G}^{*}$ level of theory. And the binding energies of the $\mathrm{C}^{\circ-} \mathrm{C}^{\circ-}$ series, $\mathrm{CH} 5^{\circ} \mathrm{CH} 5^{\circ}$ series, $\mathrm{CH} 6{ }^{\circ} \mathrm{CH} 6{ }^{\circ}$ series, $\mathrm{CH} 6^{\circ} \mathrm{CH} 5^{\circ}$ series and $\mathrm{CH} 3^{\circ} \mathrm{CH} 3^{\circ}$ series in aqueous solution are given in parentheses. ${ }^{\mathrm{a}}$

\begin{tabular}{|c|c|c|c|c|}
\hline & $\left(\mathrm{C}^{\left.\bullet-\mathrm{C}^{*}-\right)_{\mathrm{WC1}}-\mathrm{WC1}}\right.$ & $\left(C^{*-C^{*-}}\right)_{M I-W C 1}$ & $\left(\mathrm{C}^{*} \mathrm{C}^{\cdot-)_{\mathrm{MI}}-\mathrm{WC2}}\right.$ & $\left(\mathrm{C}^{\left.*-\mathrm{C}^{*-}\right)_{\mathrm{MI}-\mathrm{MI}}}\right.$ \\
\hline CS & $38.62(11.77)$ & $36.12(14.70)$ & $37.83(8.02)$ & $34.62(13.27)$ \\
\hline BS & $35.32(4.55)$ & 23.19 & $35.08(-4.45)$ & $23.57(-6.00)$ \\
\hline \multirow[t]{2}{*}{$\mathbf{T}$} & $35.32(5.00)$ & $26.44(-5.28)$ & $29.47(-7.21)$ & $23.57(-5.96)$ \\
\hline & 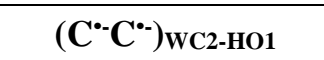 & $\left(\mathrm{C}^{\bullet-\mathrm{C}^{-0}}\right)_{\mathrm{WC2}-\mathrm{HO} 2}$ & $\left(\mathrm{C}^{\left.* \cdot \mathrm{C}^{*}-\right)_{\mathrm{N} 3-\mathrm{N} 3}}\right.$ & $\left(\mathrm{CH5}^{\circ} \mathrm{CH5}^{\circ}\right)_{\mathrm{WC1}-\mathrm{WC1}}$ \\
\hline CS & $40.95(8.82)$ & $48.63(20.56)$ & $111.47(67.43)$ & $9.93(27.21)$ \\
\hline BS & 29.76 & 27.17 & - & $-20.64(-1.378)$ \\
\hline \multirow[t]{2}{*}{$\mathbf{T}$} & $29.78(-8.72)$ & $30.38(-8.01)$ & $118.91(104.29)$ & $-20.63(-1.377)$ \\
\hline & $\left(\mathrm{CH5}^{\circ} \mathrm{CH5}^{\circ}\right)_{\mathrm{MI}-\mathrm{WC1}}$ & $\left(\mathrm{CH5}^{\circ} \mathrm{CH}^{\circ}\right)_{\mathrm{MI}-\mathrm{MI}}$ & $\left(\mathrm{CH}^{\circ} \mathrm{CH}^{\circ}\right)_{\mathrm{WC2}-\mathrm{HO}}$ & $\left(\mathrm{CH6}^{\circ} \mathrm{CH6}^{\circ}\right)_{\mathrm{WC1}-\mathrm{WC1}}$ \\
\hline CS & $7.07(27.52)$ & $8.22(27.31)$ & $10.28(25.60)$ & $8.06(23.95)$ \\
\hline BS & $-19.039(-0.168)$ & $-17.30(1.32)$ & $-11.450(-2.29)$ & $-18.33(0.35)$ \\
\hline \multirow[t]{2}{*}{$\mathbf{T}$} & $-19.040(-0.169)$ & $-16.20(1.31)$ & $-11.447(-2.29)$ & $-18.28(0.41)$ \\
\hline & $\left(\mathrm{CH6}^{\circ} \mathrm{CH6}^{\circ}\right)_{\mathrm{MI}-\mathrm{WC} 1}$ & $\left(\mathrm{CH6}^{\circ} \mathrm{CH6}^{\circ}\right)_{\mathrm{MI}-\mathrm{MI}}$ & $\left(\mathrm{CH6}^{\circ} \mathrm{CH6}^{\circ}\right)_{\mathrm{WC} 2-\mathrm{HO} 1}$ & $\left(\mathrm{CH6}^{\circ} \mathrm{CH}^{\circ}\right)_{\mathrm{N} 3-\mathrm{N} 3}$ \\
\hline CS & $-54.28(-23.20)$ & $11.43(32.76)$ & $11.60(28.32)$ & $-21.72(4.94)$ \\
\hline BS & $-17.61(1.66)$ & $-9.41(2.97)$ & $-16.332(1.247)$ & - \\
\hline \multirow[t]{2}{*}{$\mathbf{T}$} & $-17.60(1.67)$ & $-9.42(2.97)$ & $-16.330(1.248)$ & $68.60(94.00)$ \\
\hline & $\left(\mathrm{CH}^{\circ} \mathrm{CH}^{\circ}\right)_{\mathrm{WC1}-\mathrm{WC1}}$ & $\left(\mathrm{CH6}^{\circ} \mathrm{CH5}^{\circ}\right)_{\mathrm{MI}-\mathrm{WC1}}$ & $\left(\mathrm{CH}^{\circ}{ }^{\circ} \mathrm{CH}^{\circ}\right)_{\mathrm{MI}-\mathrm{MI}}$ & $\left(\mathrm{CH5}^{\circ} \mathrm{CH6}^{\circ}\right)_{\mathrm{MI}-\mathrm{WC1}}$ \\
\hline CS & - & $2.19(50.92)$ & $-27.00(-8.80)$ & $-48.49(9.45)$ \\
\hline BS & $-19.511(13.88)$ & $-18.74(61.32)$ & $-16.832(13.28)$ & - \\
\hline \multirow[t]{2}{*}{$\mathbf{T}$} & $-19.510(-0.53)$ & $-18.73(61.33)$ & $-16.826(2.06)$ & $-17.92(0.76)$ \\
\hline & $\left(\mathrm{CH5}^{\circ} \mathrm{CH}^{\circ}\right)_{\mathrm{WC} 2-\mathrm{HO} 1}$ & $\left(\mathrm{CH6}^{\circ} \mathrm{CH}^{\circ}\right)_{\mathrm{N} 3-\mathrm{N} 3}$ & $\left(\mathrm{CH} 3^{\circ} \mathrm{CH} 3^{\circ}\right)_{\mathrm{MI}-\mathrm{WC} 2}$ & $\left(\mathrm{CH}^{\circ}{ }^{\circ} \mathrm{CH} 3^{\circ}\right)_{\mathrm{MI}-\mathrm{MI}}$ \\
\hline CS & - & - & $2.97(23.29)$ & $3.74(24.02)$ \\
\hline BS & $-9.85(14.12)$ & - & $-20.61(-0.04)$ & $-20.61(0.46)$ \\
\hline \multirow[t]{2}{*}{$\mathbf{T}$} & $-9.85(-0.57)$ & $31.80(53.27)$ & $-20.61(-0.03)$ & $-20.61(0.46)$ \\
\hline & $\left(\mathrm{CH5}^{\circ} \mathrm{C}^{\circ}\right)_{\text {wC1-WC1 }}$ & $\left(\mathrm{CH5}^{\circ} \mathrm{C}^{\circ}\right)_{\text {MI-WC1 }}$ & $\left(\mathrm{CH}^{\circ} \mathrm{C}^{\circ-}\right)_{\text {MI-MI }}$ & $\left(\mathrm{CH5}^{\circ}{ }^{\circ}{ }^{\circ}\right)_{\mathrm{WC} 2-\mathrm{HO} 1}$ \\
\hline CS & -36.58 & -32.89 & -36.14 & -33.31 \\
\hline \multirow[t]{2}{*}{$\mathbf{T}$} & -32.32 & -30.73 & -25.64 & -18.92 \\
\hline & $\left(\mathrm{CH5}^{\circ} \mathrm{C}^{\circ-}\right)_{\mathrm{WC2}-\mathrm{HO} 2}$ & $\left(\mathrm{C}^{\circ-\mathrm{CH}^{\circ}}\right)_{\text {MI-WC1 }}$ & $\left(\mathrm{C}^{\circ-\mathrm{CH5}^{\circ}}\right)_{\text {MII-WC2 }}$ & $\left(\mathrm{CH6}^{\circ} \mathrm{C}^{\circ-}\right)_{\mathrm{wC1}-\mathrm{WC1}}$ \\
\hline CS & -35.08 & -41.92 & -35.85 & -72.13 \\
\hline \multirow[t]{2}{*}{$\mathbf{T}$} & -19.81 & -24.48 & -21.42 & -29.31 \\
\hline & $\left.\left(\mathrm{CH}^{\circ} \mathbf{C}^{\circ}\right)^{\circ}\right)_{\text {MI-WC1 }}$ & $\left(\mathrm{CH6}^{\circ} \mathrm{C}^{\circ-}\right)_{\text {MI-MI }}$ & $\left(\mathrm{C}^{\bullet-\mathrm{CH}^{\circ}}\right)_{\text {MI-WC1 }}$ & $\left.\left(\mathrm{CH6}^{\circ} \mathrm{C}^{\circ}\right)^{-}\right)_{\mathrm{WC} 2-\mathrm{HO}}$ \\
\hline CS & -66.57 & -72.00 & -78.31 & -72.27 \\
\hline \multirow[t]{2}{*}{$\mathbf{T}$} & -30.09 & -24.81 & -25.52 & -7.58 \\
\hline & $\left(\mathrm{CH}^{\circ} \mathrm{C}^{\circ}\right)_{\mathrm{wC1}} \mathrm{wO}^{-\mathrm{HO}}$ & $\left(\mathrm{CH6}^{\circ} \mathrm{C}^{\circ-}\right)_{\text {wC2-HO }}$ & & \\
\hline $\mathrm{CS}$ & -72.78 & -72.73 & & \\
\hline $\mathbf{T}$ & -9.13 & -8.588 & & \\
\hline
\end{tabular}

${ }^{a}$ Note: The binding energy of a di-reduced $\mathrm{CC}$ base pair is defined as $\Delta \mathrm{E}=\mathrm{E}_{\text {complex }}-\mathrm{E}_{\text {base }}-\mathrm{E}_{\text {base2 }}$, where 
$\mathrm{E}_{\text {complex }}, \mathrm{E}_{\text {base1 }}$, and $\mathrm{E}_{\text {base2 }}$ are the total energies of the base pair complex and two isolated $\mathrm{C}^{\circ-}$ and/or $\mathrm{CH} 5 \% \mathrm{CH} 6 \% \mathrm{CH} 3{ }^{\circ}$, respectively. The data in the gas phase calculated with the BSSE correction. For $\left(\mathrm{C}^{*} \mathrm{C}^{*}\right)_{\mathrm{MI}-\mathrm{WCl}},\left(\mathrm{C}^{*} \mathrm{C}^{*}\right)_{\mathrm{WC2}-\mathrm{HO} 1,}\left(\mathrm{C}^{{ }^{-} \mathrm{C}^{*}}\right)_{\mathrm{WC}-\mathrm{HO}}$, when in aqueous solution, there exists no the diradical character of the open-shell singlet state.

Table S2. Energies of the HOMO, LUMO, HOMO-LUMO (HL) gaps (in eV), and binding energies $(\mathrm{BE}$, in $\mathrm{kcal} / \mathrm{mol})$ for the $(\mathrm{CC})_{\mathrm{WC} 1-\mathrm{wC} 1},(\mathrm{CC})_{\mathrm{MI}-\mathrm{WC} 1},(\mathrm{CC})_{\mathrm{MI}-\mathrm{WC} 2},(\mathrm{CC})_{\mathrm{MI}-\mathrm{MI}},(\mathrm{CC})_{\mathrm{WC}-\mathrm{HO} 1}$, and $(\mathrm{CC})_{\mathrm{WC} 2-\mathrm{HO} 2}$ complexes.

\begin{tabular}{|l|c|c|c|c|}
\hline & HOMO & LUMO & HL gap & BE \\
\hline$(\mathrm{CC})_{\mathrm{WC1}-\mathrm{WC} 1}$ & -6.60 & -1.27 & 5.33 & -18.51 \\
\hline$(\mathrm{CC})_{\mathrm{MI}-\mathrm{WC} 1}$ & -6.28 & -1.80 & 4.48 & -20.36 \\
\hline$(\mathrm{CC})_{\mathrm{MI}-\mathrm{WC} 2}$ & -5.75 & -2.61 & 3.14 & -8.88 \\
\hline$(\mathrm{CC})_{\mathrm{MI}-\mathrm{MI}}$ & -6.59 & -1.58 & 5.01 & -20.64 \\
\hline$(\mathrm{CC})_{\mathrm{WC2}-\mathrm{HO} 1}$ & -5.77 & -2.62 & 3.15 & -9.71 \\
\hline$(\mathrm{CC})_{\mathrm{WC2}-\mathrm{HO} 2}$ & -5.77 & -2.87 & 2.90 & -8.66 \\
\hline
\end{tabular}

Table S3. The NPA charges on the two cytosine moieties in the $(\mathrm{CC})^{2-} \mathrm{WC}^{-\mathrm{WC} 1},(\mathrm{CC})^{2-} \mathrm{MI}-\mathrm{WC}$, $(\mathrm{CC})^{2-}{ }_{\mathrm{MI}-\mathrm{WC} 2},(\mathrm{CC})^{2-}{ }_{\mathrm{MI}-\mathrm{MI}},(\mathrm{CC})^{2-}{ }_{\mathrm{WC} 2-\mathrm{HO} 1}$, and $(\mathrm{CC})^{2-}{ }_{\mathrm{WC} 2-\mathrm{HO} 2}$ complexes in aqueous solution.

\begin{tabular}{|c|c|c|c|c|c|}
\hline$(\mathrm{CC})^{2-}$ & \multicolumn{2}{|c|}{$\begin{array}{c}\mathrm{NPA} \\
\mathrm{C} 1 \mid \mathrm{C} 2\end{array}$} & $(\mathrm{CC})^{2-}$ & \multicolumn{2}{|c|}{$\begin{array}{c}\text { NPA } \\
\mathrm{C} 1 \mid \mathrm{C} 2\end{array}$} \\
\hline$(\mathrm{CC})^{2-}$ wC1-wC1 & -1.00 & -1.00 & $(\mathrm{CC})^{2-}$ MI-MI & -1.00 & -1.00 \\
\hline$(\mathrm{CC})^{2-} \mathrm{MI}-\mathrm{WC1}$ & -0.94 & -1.06 & $(\mathrm{CC})^{2-}{ }^{2-} 2-\mathrm{HO} 1$ & -1.65 & -0.35 \\
\hline$(\mathrm{CC})^{2-}$ MI-WC2 & -0.68 & -1.32 & $(\mathrm{CC})^{2-}{ }_{\mathrm{WC} 2-\mathrm{HO} 2}$ & -1.06 & $\begin{array}{l}-0.94 \\
\end{array}$ \\
\hline
\end{tabular}


Table S4. Occupation number of HOMO and LUMO, number of electrons outside closed-shell bonding orbitals (BO) and diradical percentages (\%) calculated using CASSCF method.

\begin{tabular}{|c|c|c|c|c|}
\hline $\mathrm{C}^{\bullet \cdot-\mathrm{C}^{\bullet-}}$ & HOMO & LUMO & $\begin{array}{c}\text { Total occupancy } \\
\text { outside BO } \\
\end{array}$ & $\begin{array}{c}\text { Diradical } \\
\text { percentage }\end{array}$ \\
\hline$\left(C^{\cdot-C^{*-}}\right)$ wC1-WC1 & 0.992 & 1.008 & 1.190 & 100 \\
\hline$\left(\mathrm{C}^{\circ-\mathrm{C}^{\circ-}}\right)_{\mathrm{MI}-\mathrm{WC} 2}$ & 1.060 & 0.940 & 1.148 & 94.0 \\
\hline$\left(C^{\bullet--} C^{\bullet-}\right)_{\text {MI-MI }}$ & 1.000 & 1.000 & 1.217 & 100 \\
\hline $\mathrm{CH5}^{\circ} \mathrm{CH5}^{\circ}$ & HOMO & LUMO & $\begin{array}{c}\text { Total occupancy } \\
\text { outside BO } \\
\end{array}$ & $\begin{array}{c}\text { Diradical } \\
\text { percentage }\end{array}$ \\
\hline$\left(\mathrm{CH5}^{\circ} \mathrm{CH5}^{\circ}\right)$ wC1-WC1 & 1.003 & 1.000 & 1.175 & 100 \\
\hline$\left(\mathrm{CH5}^{\circ} \mathrm{CH5}^{\circ}\right)_{\text {MI-WC1 }}$ & 1.016 & 0.985 & 1.124 & 98.5 \\
\hline$\left(\mathrm{CH5}^{\circ} \mathrm{CH5}^{\circ}\right)_{\text {MI-MI }}$ & 1.025 & 0.977 & 1.165 & 97.7 \\
\hline$\left(\mathrm{CH5}^{\circ} \mathrm{CH5}^{\circ}\right)$ WC2-HO1 & 1.042 & 0.960 & 1.151 & 96.0 \\
\hline $\mathrm{CH6}^{\circ} \mathrm{CH6}^{\circ}$ & HOMO & LUMO & $\begin{array}{c}\text { Total occupancy } \\
\text { outside BO }\end{array}$ & $\begin{array}{l}\text { Diradical } \\
\text { percentage }\end{array}$ \\
\hline$\left(\mathrm{CH6}^{\circ} \mathrm{CH} 6^{\circ}\right)$ wC1-WC1 & 1.038 & 0.971 & 1.063 & 97.1 \\
\hline$\left(\mathrm{CH6}^{\circ} \mathrm{CH6}^{\circ}\right)^{\text {MI-WC1 }}$ & 1.013 & 0.995 & 1.200 & 99.5 \\
\hline$\left(\mathrm{CH6}^{\circ} \mathrm{CH6}^{\circ}\right)_{\text {MI-MI }}$ & 1.007 & 1.000 & 1.175 & 100 \\
\hline$\left(\mathrm{CH6}^{\circ} \mathrm{CH6}^{\circ}\right)$ wC2-HO1 & 1.027 & 0.980 & 1.147 & 98.0 \\
\hline $\mathrm{CH6}^{\circ} \mathrm{CH5}^{\circ}$ & HOMO & LUMO & $\begin{array}{c}\text { Total occupancy } \\
\text { outside BO }\end{array}$ & $\begin{array}{c}\text { Diradical } \\
\text { percentage }\end{array}$ \\
\hline$\left(\mathrm{CH6}^{\circ} \mathrm{CH5}^{\circ}\right)$ wC1-wC1 & 1.006 & 0.998 & 1.150 & 99.8 \\
\hline$\left(\mathrm{CH6}^{\circ} \mathrm{CH5}^{\circ}\right)_{\mathrm{MI}-\mathrm{WC1}}$ & 1.005 & 1.000 & 1.197 & 100 \\
\hline$\left(\mathrm{CH6}^{\circ} \mathrm{CH5}^{\circ}\right)_{\mathrm{MI}-\mathrm{MI}}$ & 1.010 & 0.994 & 1.166 & 99.4 \\
\hline$\left(\mathrm{CH5}^{\circ} \mathrm{CH6}^{\circ}\right){ }_{\mathrm{WC} 2-\mathrm{HO} 1}$ & 0.997 & 1.007 & 1.197 & 100 \\
\hline $\mathrm{CH}^{\circ} \mathrm{CH}^{\circ}$ & HOMO & LUMO & $\begin{array}{c}\text { Total occupancy } \\
\text { outside BO } \\
\end{array}$ & $\begin{array}{c}\text { Diradical } \\
\text { percentage }\end{array}$ \\
\hline$\left(\mathrm{CH}^{\circ}{ }^{\circ} \mathrm{CH} 3^{\circ}\right)_{\mathrm{MI}-W C 2}$ & 0.994 & 1.006 & 1.196 & 100 \\
\hline$\left(\mathrm{CH3}^{\circ} \mathrm{CH} 3^{\circ}\right)_{\mathrm{MI}-\mathrm{MI}}$ & 1.004 & 0.995 & 1.194 & 99.5 \\
\hline
\end{tabular}


Table S5. Occupation number of LUMO, number of electrons outside closed-shell bonding orbitals (BO) and diradical percentages (\%) calculated using CASSCF method.

\begin{tabular}{|c|c|c|c|}
\hline$\left(\mathrm{CH5}^{\circ} \mathrm{CH5}^{\circ}\right)_{\pi \pi}$ & LUMO & $\begin{array}{c}\text { Total occupancy outside } \\
\text { BO } \\
\end{array}$ & $\begin{array}{c}\text { Diradical } \\
\text { percentage }\end{array}$ \\
\hline$\left(\mathrm{CH5}^{\circ} \mathrm{CH5}^{\circ}\right)^{\mathrm{cr}}{ }_{\pi \pi}$ & 0.976 & 1.140 & 97.6 \\
\hline$\left(\text { CH5 }^{\circ} \mathrm{CH5}^{\circ}\right)^{\mathrm{ap}}{ }_{\pi \pi}$ & 0.973 & 1.159 & 97.3 \\
\hline$\left(\mathrm{CH5}^{\circ} \mathrm{CH5}^{\circ}\right)^{\mathrm{cl}} \pi \pi$ & 0.975 & 1.140 & 97.5 \\
\hline$\left(\mathrm{CH6}^{\circ} \mathrm{CH6}^{\circ}\right)_{\pi \pi}$ & LUMO & $\begin{array}{c}\text { Total occupancy outside } \\
\text { BO } \\
\end{array}$ & $\begin{array}{c}\text { Diradical } \\
\text { percentage }\end{array}$ \\
\hline$\left(\mathrm{CH6}^{\circ} \mathrm{CH6}^{\circ}\right)^{\mathrm{cr}} \pi \pi$ & 0.870 & 1.083 & 87.0 \\
\hline$\left(\mathrm{CH6}^{\circ} \mathrm{CH6}^{\circ}\right)^{\text {ap }}{ }_{\pi \pi}$ & 0.998 & 1.210 & 99.8 \\
\hline$\left(\mathrm{CH6}^{\circ} \mathrm{CH6}^{\circ}\right)^{\mathrm{cl}} \pi \pi$ & 0.870 & 1.083 & 87.0 \\
\hline$\left(\mathrm{CH5}^{\circ} \mathrm{CH6}^{\circ}\right)_{\pi \pi}$ & LUMO & $\begin{array}{c}\text { Total occupancy outside } \\
\text { BO } \\
\end{array}$ & $\begin{array}{c}\text { Diradical } \\
\text { percentage }\end{array}$ \\
\hline$\left(\mathrm{CH5}^{\circ} \mathrm{CH6}^{\circ}\right)^{\mathrm{cr}}{ }_{\pi \pi}$ & 0.905 & 1.112 & 90.5 \\
\hline$\left(\mathrm{CH5}^{\circ} \mathrm{CH6}^{\circ}\right)^{\mathrm{ap}} \mathrm{p}_{\pi \pi}$ & 0.910 & 1.109 & 91.0 \\
\hline$\left(\mathrm{CH3}^{\circ} \mathrm{CH} 3^{\circ}\right)_{\pi \pi}$ & LUMO & $\begin{array}{c}\text { Total occupancy outside } \\
\text { BO } \\
\end{array}$ & $\begin{array}{c}\text { Diradical } \\
\text { percentage }\end{array}$ \\
\hline$\left(\mathrm{CH3}^{\circ} \mathrm{CH3}^{\circ}\right)^{\mathrm{cr}} \pi \pi$ & 0.707 & 0.898 & 70.7 \\
\hline$\left(\mathrm{CH3}^{\circ} \mathrm{CH3}^{\circ}\right)^{\text {ap }}{ }_{\pi \pi}$ & 0.888 & 1.073 & 88.8 \\
\hline$\left(\mathrm{CH3}^{\circ} \mathrm{CH3}^{\circ}\right)^{\mathrm{cl}}{ }_{\pi \pi}$ & 0.707 & 0.898 & 70.7 \\
\hline
\end{tabular}

Table S6. The Calculated Energies (in a.u.) and $\left\langle S^{2}\right\rangle$ Values, and Intermolecular Magnetic Exchange Coupling Constants $J$, and Energy Order of the $\left(\mathrm{CH} 5^{\circ} \mathrm{CH} 5^{\circ}\right)_{\pi \pi}$ Base Pairs Calculated at the MP2/6-311++G**//M06-2X/6-311+G* Level.

\begin{tabular}{|c|c|c|c|l|}
\hline$\left(\mathrm{CH} 5{ }^{\circ} \mathrm{CH} 5^{\circ}\right)_{\pi \pi}$ & $\mathbf{E}_{\mathbf{T}}\left(<\mathrm{S}^{2}>\right)$ & $\mathbf{E}_{\mathbf{B S}}\left(<\mathbf{S}^{2}>\right)$ & $\boldsymbol{J}\left(\mathbf{c m}^{-1}\right)$ & Energy order \\
\hline$\left(\mathrm{CH} 5^{\circ} \mathrm{CH} 5^{\circ}\right)^{\mathrm{cr}}{ }_{\pi \pi}$ & $-789.055007(2.03)$ & $-789.054461(1.01)$ & 117.35 & $\mathrm{E}_{\mathrm{T}}<\mathrm{E}_{\mathrm{BS}}<\mathrm{E}_{\mathrm{CS}}$ \\
\hline$\left(\mathrm{CH} 5^{\circ} \mathrm{CH} 5^{\circ}\right)^{\mathrm{ap}}{ }_{\pi \pi}$ & $-789.0621064(2.03)$ & $-789.0621445(1.03)$ & -8.36 & $\mathrm{E}_{\mathrm{BS}}<\mathrm{E}_{\mathrm{T}}<\mathrm{E}_{\mathrm{CS}}$ \\
\hline$\left(\mathrm{CH} 5^{\circ} \mathrm{CH} 5^{\circ}\right)^{\mathrm{cl}}{ }_{\pi \pi}$ & $-790.883722(2.03)$ & $-790.8829026(1.03)$ & 126.68 & $\mathrm{E}_{\mathrm{T}}<\mathrm{E}_{\mathrm{BS}}<\mathrm{E}_{\mathrm{CS}}$ \\
\hline
\end{tabular}


Table S7. The Calculated Energies (a.u.) and $\left\langle\mathrm{S}^{2}\right\rangle$ of the $\mathrm{CH} 5^{\circ} \mathrm{CH} 5^{\circ}$ series, $\mathrm{CH} 6^{\circ} \mathrm{CH} 6^{\circ}$ series, $\mathrm{CH} 5^{\circ} \mathrm{CH} 6^{\circ}$ series and $\mathrm{CH} 3^{\circ} \mathrm{CH} 3^{\circ}$ series in the aqueous solution.

\begin{tabular}{|c|c|c|c|}
\hline $\mathrm{CH5}^{\circ} \mathrm{CH} 5^{\circ}$ & Ecs & $\operatorname{EBS}_{B S}\left(\left\langle\mathbf{S}^{2}>\right)\right.$ & $\mathbf{E}_{\mathbf{T}}\left(\left\langle\mathbf{S}^{2}\right\rangle\right)$ \\
\hline$\left(\mathrm{CH} 5^{\circ} \mathrm{CH} 5^{\circ}\right)_{\mathrm{wC1}-\mathrm{wC} 1}$ & -791.2572639 & $-791.3028234(1.01)$ & $-791.2175628(2.01)$ \\
\hline$\left(\mathrm{CH} 5^{\circ} \mathrm{CH} 5^{\circ}\right)_{\mathrm{MI}-\mathrm{WC} 1}$ & -791.256763 & $-791.3008952(1.01)$ & $-791.3008965(2.01)$ \\
\hline$\left(\mathrm{CH}^{\circ} \mathrm{CH} 5^{\circ}\right)_{\mathrm{MI}-\mathrm{MI}}$ & -791.2571093 & $-791.2119905(1.01)$ & $-791.2119702(2.01)$ \\
\hline$\left(\mathrm{CH} 5^{\circ} \mathrm{CH} 5^{\circ}\right)_{\mathrm{WC} 2-\mathrm{HO} 1}$ & -791.2598252 & $-791.202259(1.01)$ & $-791.2022527(2.01)$ \\
\hline $\mathrm{CH6}^{\circ} \mathrm{CH6}^{\circ}$ & ECS & $\mathbf{E}_{B S}\left(\left\langle\mathbf{S}^{2}>\right)\right.$ & $\mathbf{E}_{\mathbf{T}}\left(\left\langle\mathbf{S}^{2}\right\rangle\right)$ \\
\hline$\left(\mathrm{CH}^{\circ}{ }^{\circ} \mathrm{CH} 6^{\circ}\right)_{\mathrm{WC1}-\mathrm{wC} 1}$ & -791.275591 & $-791.3132062(1.01)$ & $-791.3131077(2.02)$ \\
\hline$\left(\mathrm{CH6}^{\circ} \mathrm{CH} 6^{\circ}\right)_{\mathrm{MI}-\mathrm{WC} 1}$ & -791.350731 & $-791.3111113(1.01)$ & $-791.3111032(2.01)$ \\
\hline$\left(\mathrm{CH}^{\circ} \mathrm{CH}^{\circ}\right)_{\mathrm{MI}-\mathrm{MI}}$ & -791.261553 & $-791.3090277(1.02)$ & $-791.3090276(2.02)$ \\
\hline$\left(\mathrm{CH}^{\circ}{ }^{\circ} \mathrm{CH} 6^{\circ}\right)_{\mathrm{WC2}-\mathrm{HO} 1}$ & -791.268627 & $-791.3117739(1.01)$ & $-791.3117725(2.01)$ \\
\hline$\left(\mathrm{CH}^{\circ}{ }^{\circ} \mathrm{CH}^{\circ}\right)_{\mathrm{N} 3-\mathrm{N} 3}$ & -791.305889 & - & $-791.1639619(2.01)$ \\
\hline $\mathrm{CH6}^{\circ} \mathrm{CH5}^{\circ}$ & $\mathbf{E}_{\mathrm{CS}}$ & $\operatorname{E}_{B S}\left(\left\langle\mathbf{S}^{2}\right\rangle\right)$ & $\mathbf{E}_{\mathrm{T}}\left(\left\langle\mathbf{S}^{2}>\right)\right.$ \\
\hline$\left(\mathrm{CH}^{\circ} \mathrm{CH} 5^{\circ}\right)_{\mathrm{WC1}-\mathrm{wC} 1}$ & - & $-791.2850774(0.28)$ & $-791.3080313(2.01)$ \\
\hline$\left(\mathrm{CH} 6^{\circ} \mathrm{CH} 5^{\circ}\right)_{\mathrm{MI}-\mathrm{WC} 1}$ & -791.306023 & $-791.2860332(0.19)$ & $-791.2426345(2.01)$ \\
\hline$\left(\mathrm{CH}^{\circ} \mathrm{CH} 5^{\circ}\right)_{\mathrm{MI}-\mathrm{MI}}$ & -791.321217 & $-791.286028(0.17)$ & $-791.3039036(2.02)$ \\
\hline$\left(\mathrm{CH} 5^{\circ} \mathrm{CH} 6^{\circ}\right)_{\mathrm{MI}-\mathrm{WC} 1}$ & -791.292119 & - & $-791.2114183(2.01)$ \\
\hline$\left(\mathrm{CH} 5^{\circ} \mathrm{CH} 6^{\circ}\right)_{\mathrm{WC} 2-\mathrm{HO} 1}$ & - & $-791.2846995(0.34)$ & $-791.3081043(2.01)$ \\
\hline$\left(\mathrm{CH}^{\circ} \mathrm{CH} 6^{\circ}\right)_{\mathrm{N} 3-\mathrm{N} 3}$ & - & - & $-791.2223022(2.01)$ \\
\hline $\mathrm{CH3}^{\circ} \mathrm{CH3}^{\circ}$ & $\mathbf{E}_{\mathrm{CS}}$ & $\left.\mathbf{E}_{B S}\left(<\mathbf{S}^{2}\right\rangle\right)$ & $\mathbf{E}_{\mathbf{T}}\left(\left\langle\mathbf{S}^{2}\right\rangle\right)$ \\
\hline$\left(\mathrm{CH} 3^{\circ} \mathrm{CH} 3^{\circ}\right)_{\mathrm{MI}-\mathrm{WC} 2}$ & -791.1934351 & $-791.2309923(1.03)$ & $-791.2309896(2.03)$ \\
\hline$\left(\mathrm{CH} 3^{\circ} \mathrm{CH} 3^{\circ}\right)_{\mathrm{MI}-\mathrm{MI}}$ & -791.1920785 & $-791.2308794(1.03)$ & $-791.2308775(2.04)$ \\
\hline
\end{tabular}


$\mathrm{C}$

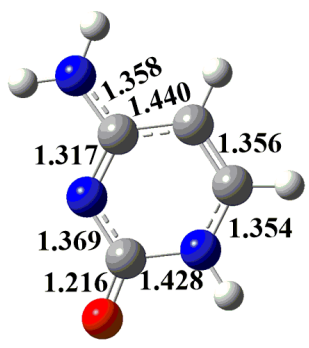

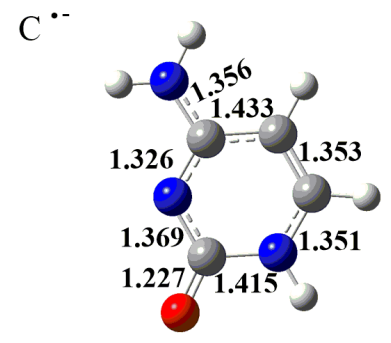
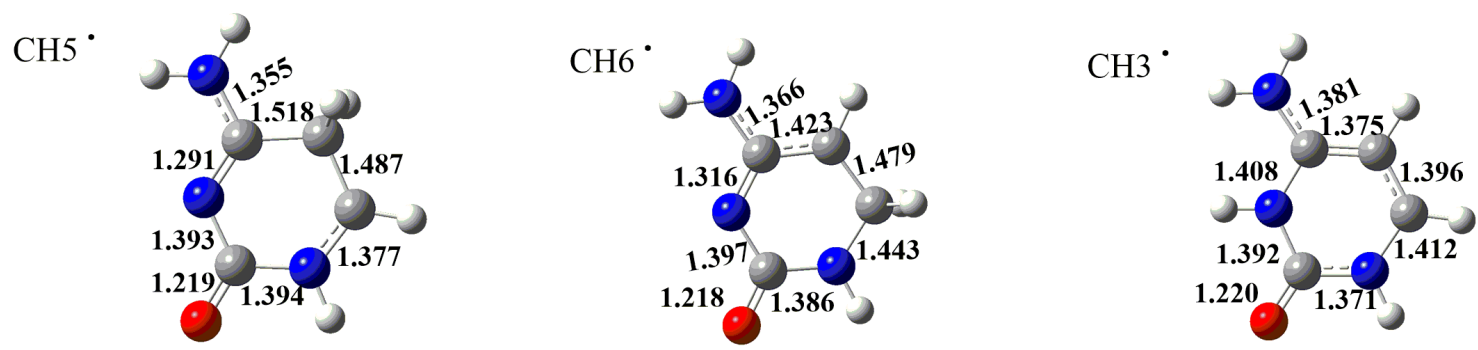

Figure S1. Optimized structures of the C, with their main geometrical parameters. The selected bond lengths are in $\AA$

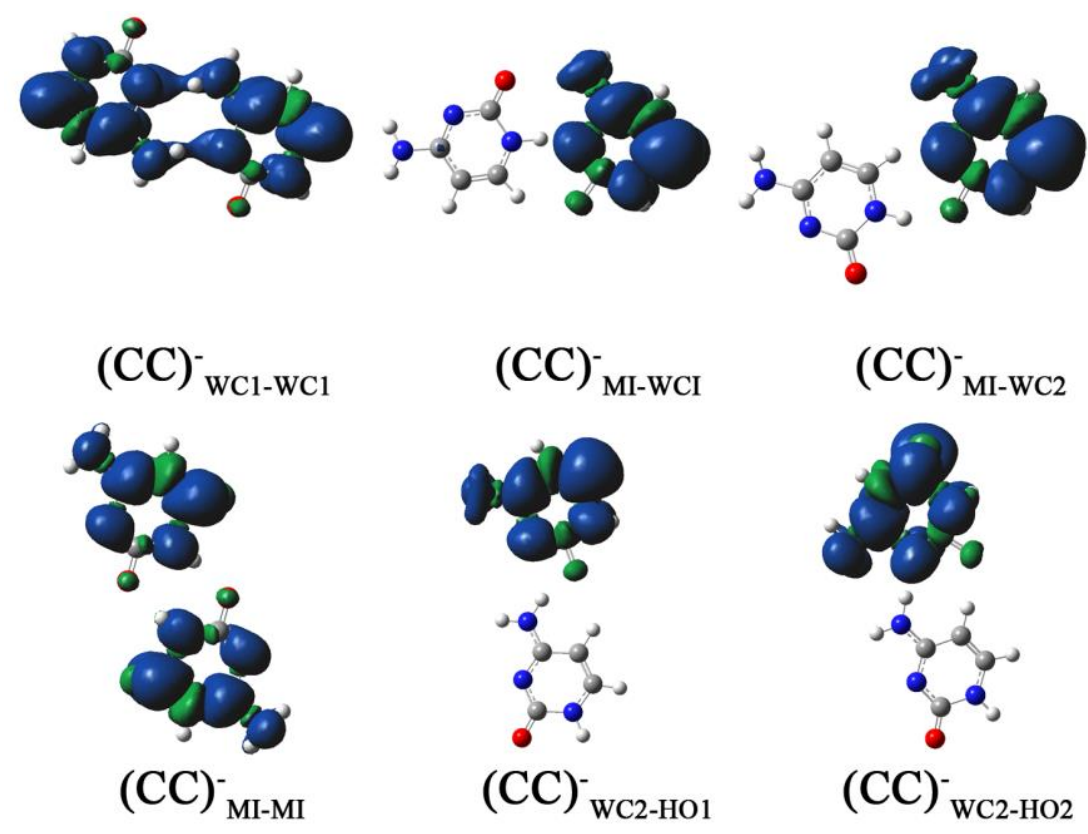

Figure S2. The spin density distributions for the single-electron reduced (CC) ${ }^{-}{ }^{-} 1-\mathrm{WC}$, $(\mathrm{CC})^{-} \mathrm{MI}^{-\mathrm{WC} 1},(\mathrm{CC})^{-} \mathrm{MI}^{-\mathrm{WC} 2},(\mathrm{CC})^{-} \mathrm{MI}-\mathrm{MI},(\mathrm{CC})^{-} \mathrm{WC}^{-\mathrm{HO} 1}$ and $(\mathrm{CC})^{-}{ }^{-} \mathrm{WC} 2-\mathrm{HO} 2$. 
I) $\left(\mathrm{C}^{\left.\bullet-\mathrm{C}^{\bullet-}\right) \text { series }}\right.$

CS

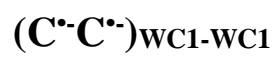

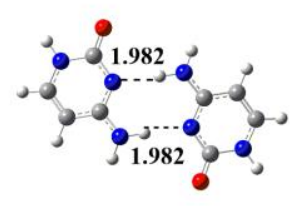

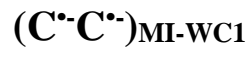

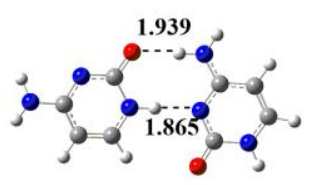

$\left(\mathrm{C}^{\circ \cdot} \mathrm{C}^{\bullet-}\right)_{\mathrm{MII}-\mathrm{WC2}}$

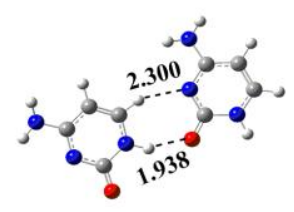

$\left(\mathrm{C}^{\bullet-\mathrm{C}^{\bullet--}}\right)_{\mathrm{MI}-\mathrm{MI}}$
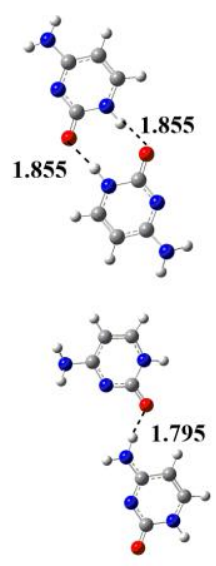

$\left(\mathrm{C}^{\left.\circ-\mathrm{C}^{\circ-}\right) \text { WC2-HO1 }}\right.$

$\left(\mathrm{C}^{\circ} \mathrm{C}^{-*}\right)_{\mathrm{WC2}-\mathrm{HO} 2}$
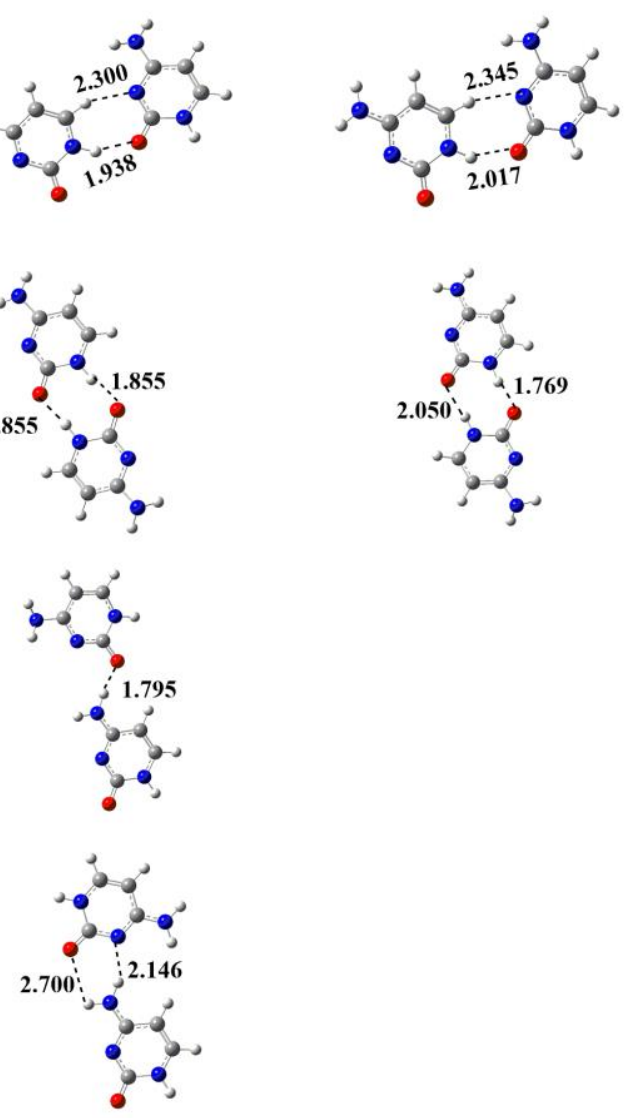

$\left(\mathrm{C}^{\bullet-\mathrm{C}^{\bullet--}}\right)_{\mathrm{N3}-\mathrm{N3}}$

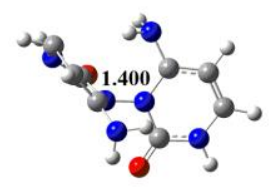

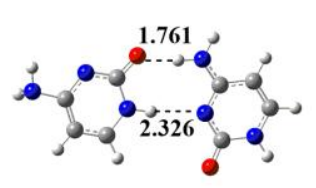
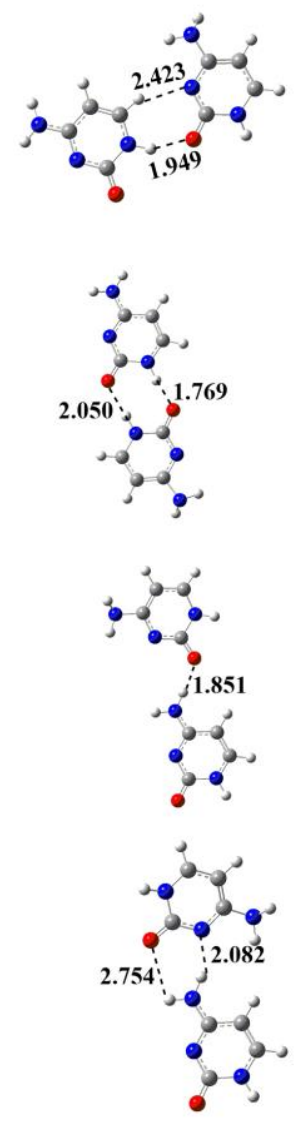

BS
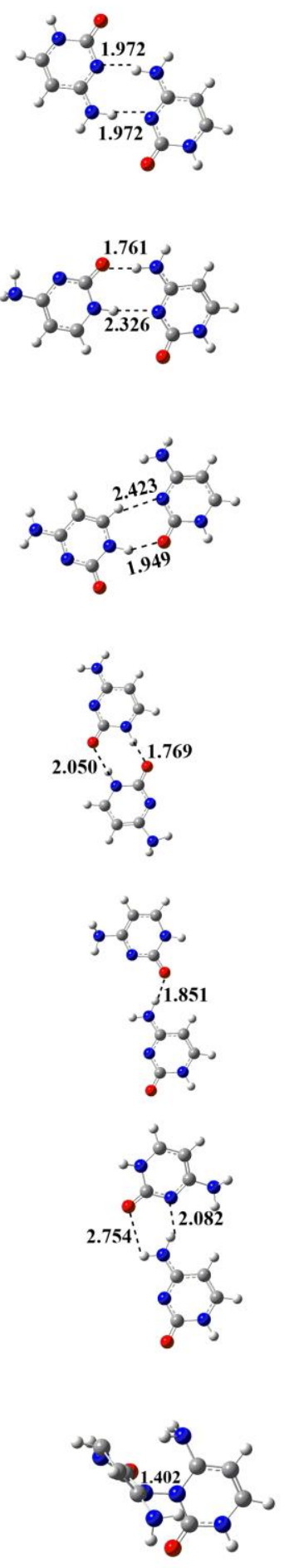

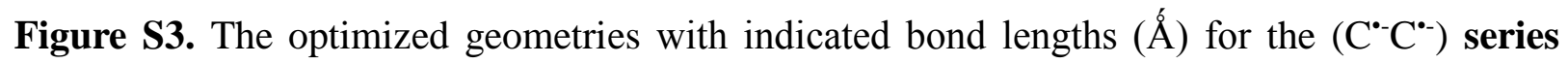
base pairs (CS. closed-shell singlet; BS. open-shell broken-symmetry singlet; T. triplet state).

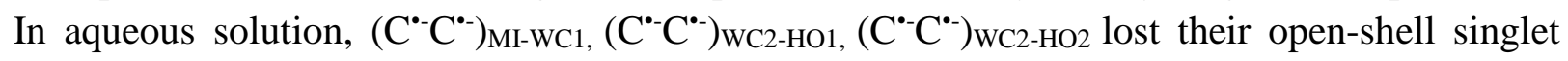
states. 


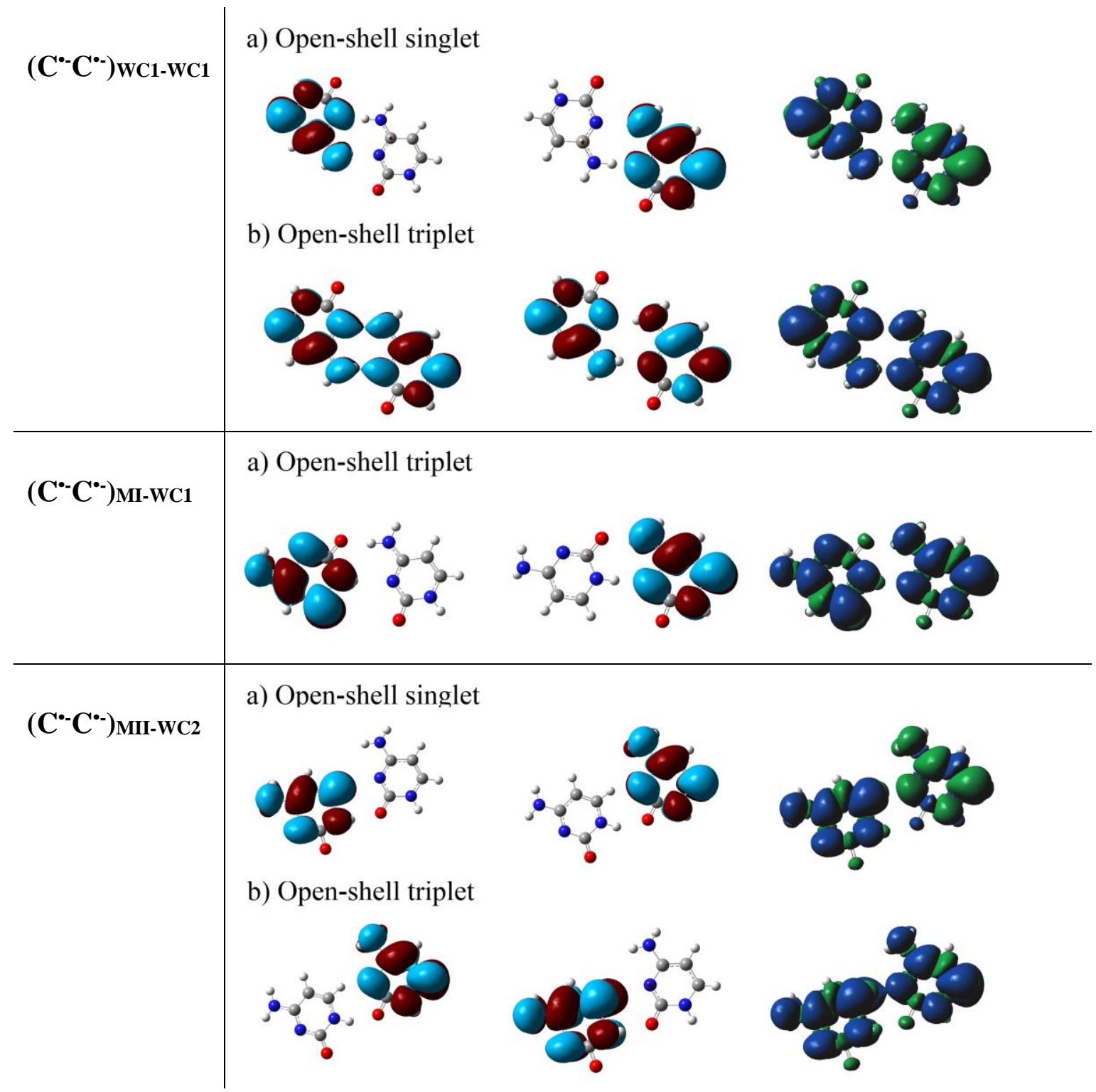

Figure S4. Singly occupied molecular orbitals (SOMOs) and spin density surfaces for open-shell broken-symmetry (BS) singlet and triplet $(\mathrm{T})$ states of the $\left(\mathrm{C}^{\circ-} \mathrm{C}^{*-}\right) \mathrm{WC1}-\mathrm{WC}$,

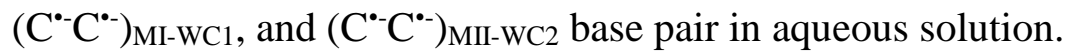




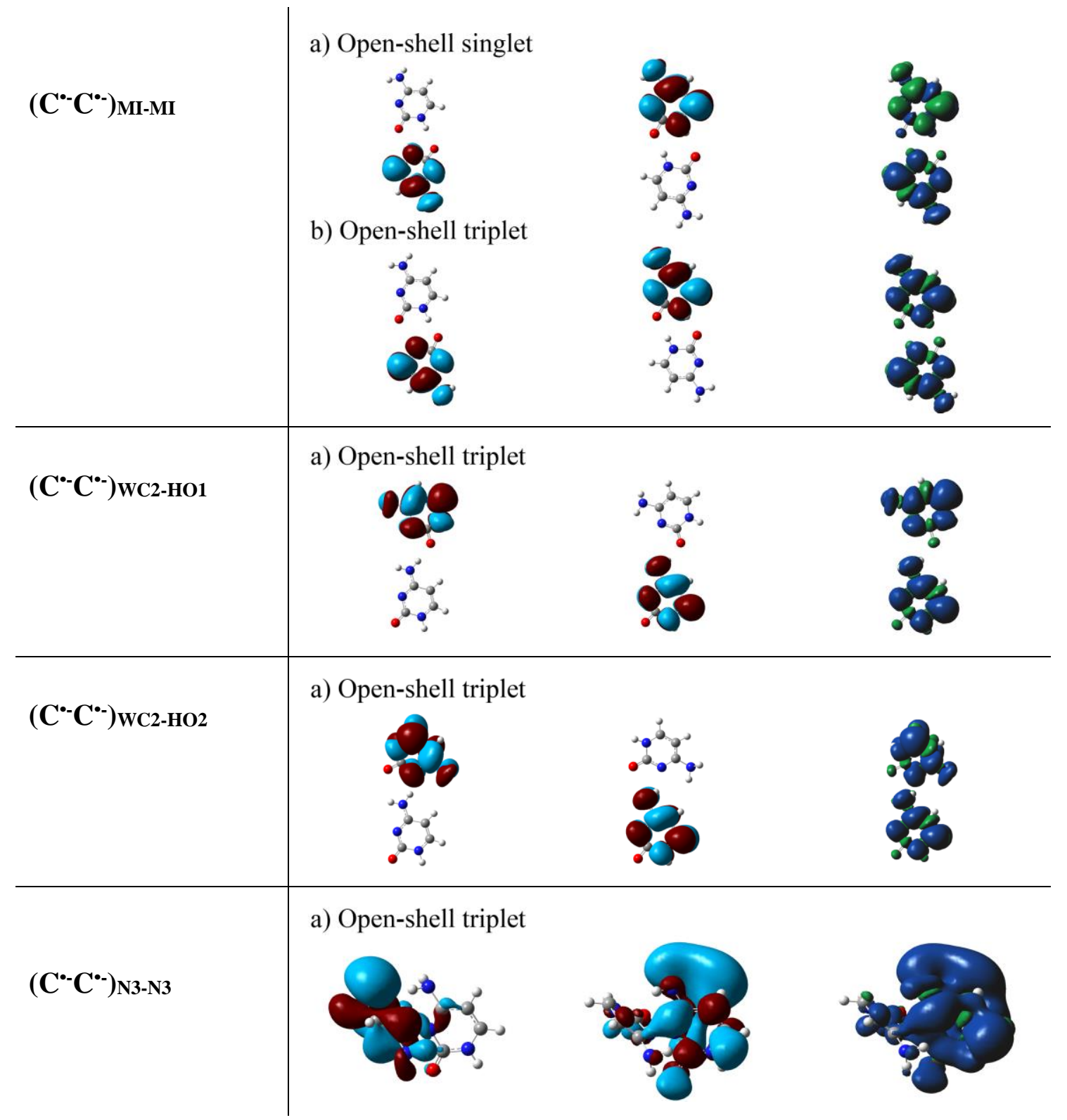

Figure S5. Singly occupied molecular orbitals (SOMOs) and spin density surfaces for

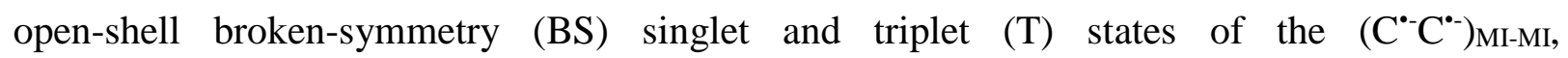

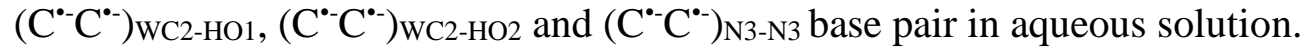


II) $\left(\mathrm{CH}^{\circ} \mathrm{CH5}^{\circ}\right)$ series

CS

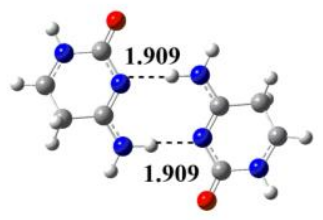

$\left(\mathrm{CH5}^{\circ} \mathrm{CH5}^{\circ}\right)_{\mathrm{MI}-\mathrm{WC1}}$
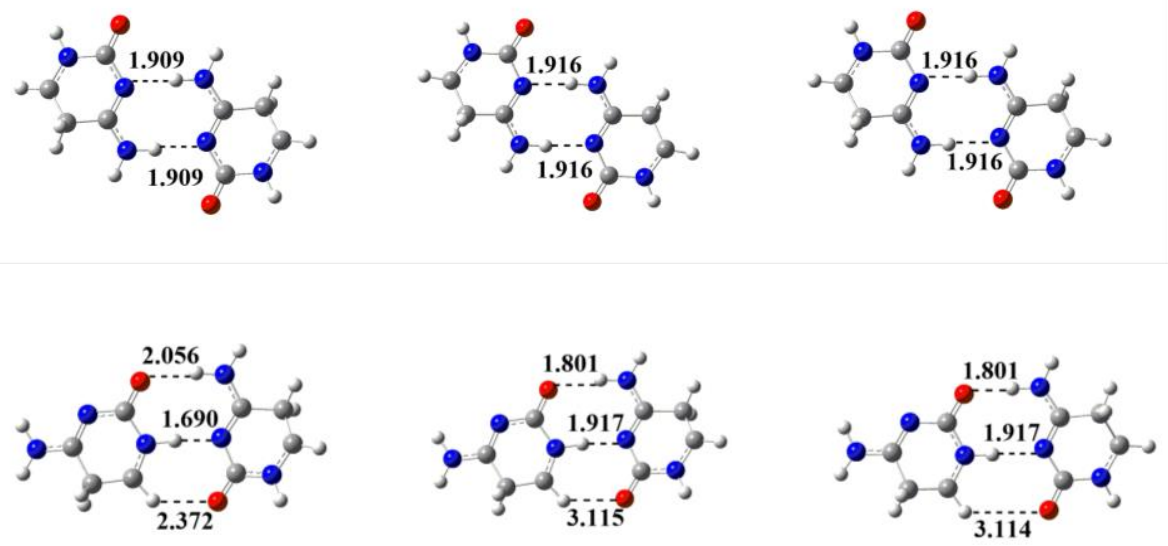

$\left(\mathrm{CH5}^{\circ} \mathrm{CH5}^{\circ}\right)_{\mathrm{MI}-\mathrm{MI}}$

$\left(\mathrm{CH5}^{\circ} \mathrm{CH5}^{\circ}\right){ }^{\mathrm{wC2}} \mathrm{HO1}^{-\mathrm{HO}}$
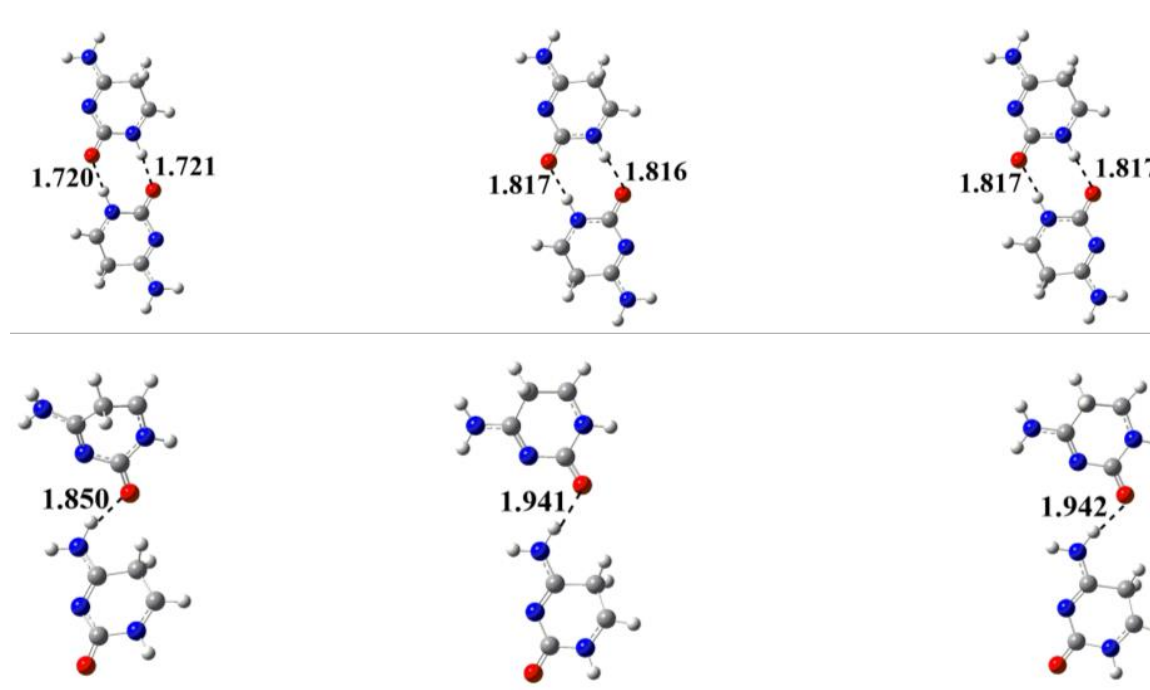

Figure S6. The optimized geometries with indicated bond lengths ( $⿱$ Á) for the $\mathrm{CH} 5^{\circ} \mathrm{CH} 5^{\circ}$ series base pairs (a. closed-shell singlet; b. open-shell broken-symmetry singlet; c. triplet state). 
a) Open-shell singlet

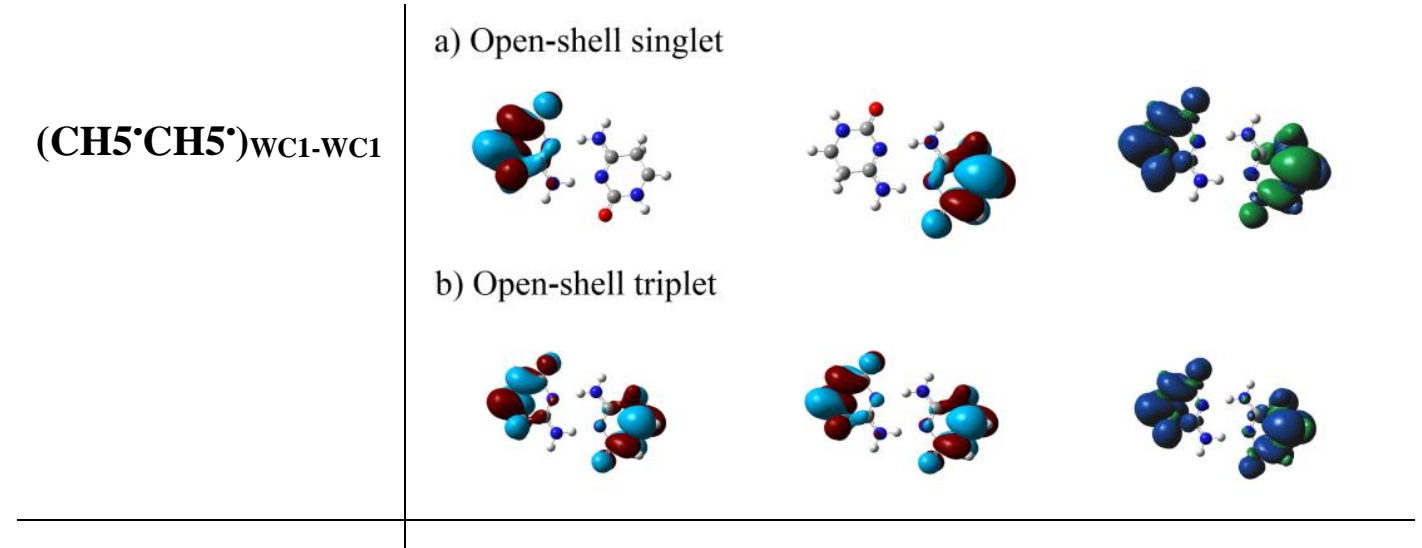

a) Open-shell singlet

\section{$\left(\mathrm{CH5}^{\circ} \mathrm{CH5}^{\circ}\right)_{\mathrm{MI}-\mathrm{WC1}}$}
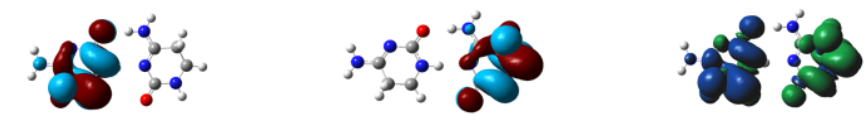

b) Open-shell triplet
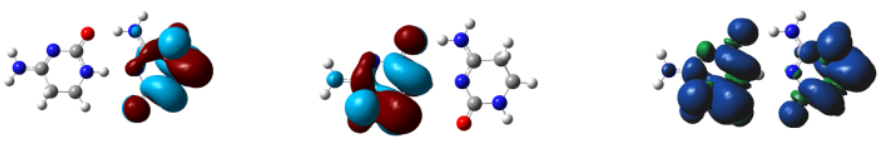

a) Open-shell singlet

(CH5 $\left.^{\circ} \mathrm{CH5}^{\circ}\right)_{\mathrm{MI}-\mathrm{MI}}$

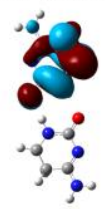

b) Open-shell triplet
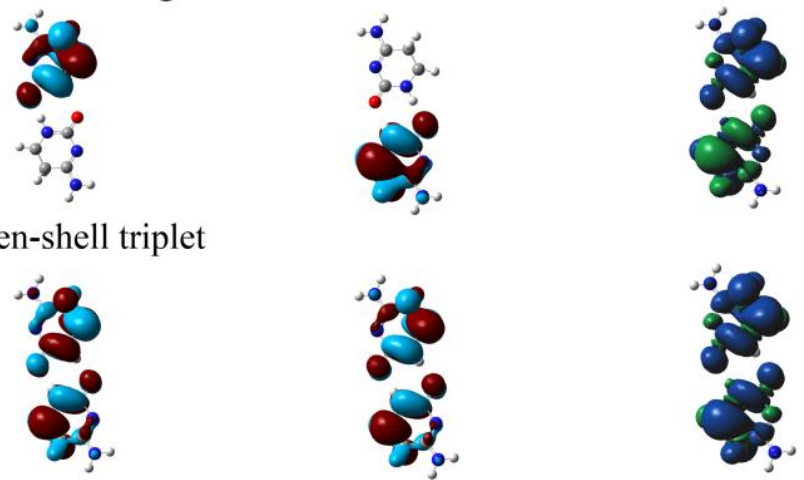

a) Open-shell singlet

$\left(\mathrm{CH5}^{\circ} \mathrm{CH5}^{\circ}\right)_{\mathrm{WC} 2-\mathrm{HO1}}$
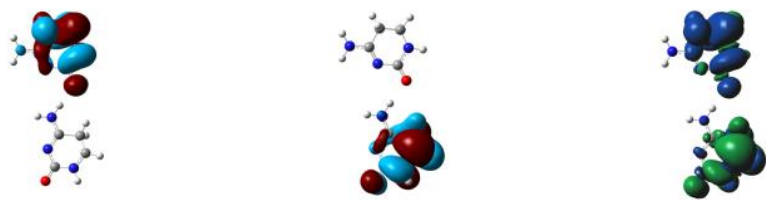

b) Open-shell triplet
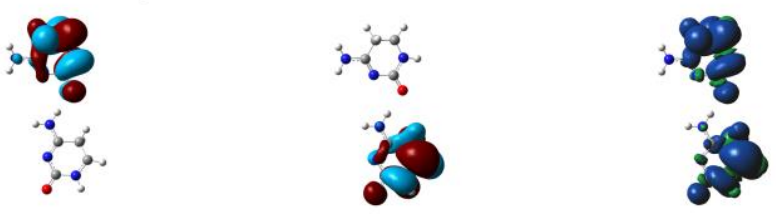

Figure S7. Singly occupied molecular orbitals (SOMOs) and spin density surfaces for open-shell broken-symmetry (BS) singlet and triplet (T) states of the $\left(\mathrm{CH} 5^{\circ} \mathrm{CH} 5^{\circ}\right) \mathrm{WC1}$-WC1 , $\left(\mathrm{CH} 5^{\circ} \mathrm{CH} 5^{\circ}\right)_{\mathrm{MI}-\mathrm{WC} 1},\left(\mathrm{CH} 5^{\circ} \mathrm{CH} 5^{\circ}\right)_{\mathrm{MI}-\mathrm{MI}}$ and $\left(\mathrm{CH} 5^{\circ} \mathrm{CH} 5^{\circ}\right)_{\mathrm{WC} 2-\mathrm{HO} 1}$ base pair. 
III) $\left(\mathrm{CH6}^{\circ} \mathrm{CH6}^{\circ}\right)$ series

CS

$\left(\mathrm{CH6}^{\circ} \mathrm{CH}^{\circ}\right)^{\text {wC1-wC1 }}$

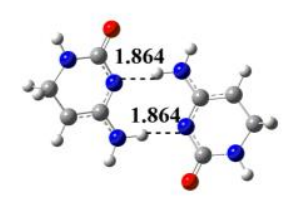

$\left(\mathrm{CH6}^{\circ} \mathrm{CH6}^{\circ}\right)_{\mathrm{MI}-\mathrm{WC1}}$
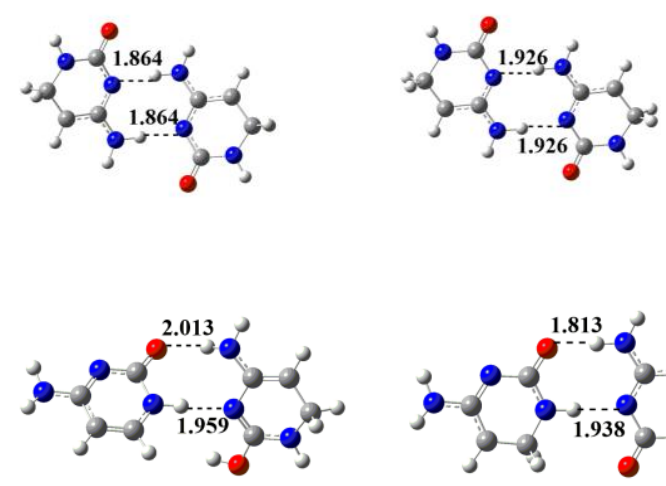

BS

$\mathrm{T}$
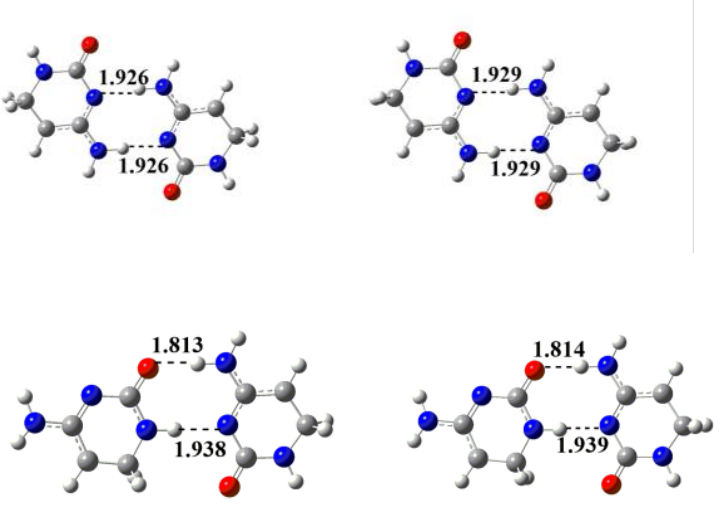

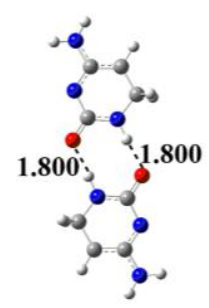

$\left(\mathrm{CH6}^{\circ} \mathrm{CH6}^{\circ}\right)_{\mathrm{MI}-\mathrm{MI}}$

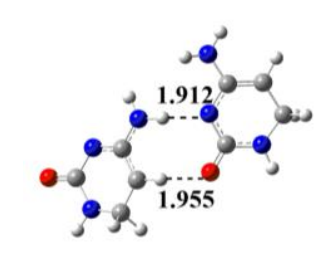

$\left(\mathrm{CH6}^{\circ} \mathrm{CH6}^{\circ}\right)^{\mathrm{wC2}-\mathrm{HO} 1}$
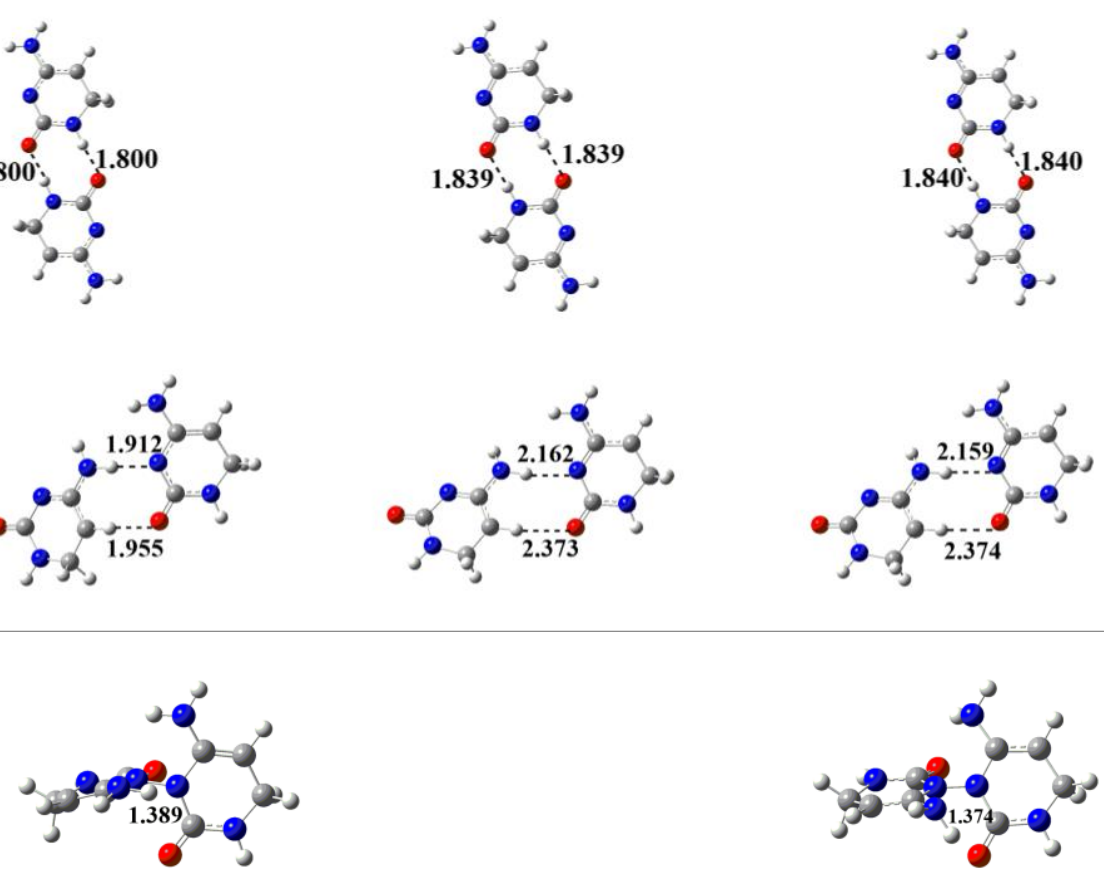

$\left(\mathrm{CH6}^{\circ} \mathrm{CH6}^{\circ}\right)_{\mathrm{N} 3-\mathrm{N3}}$

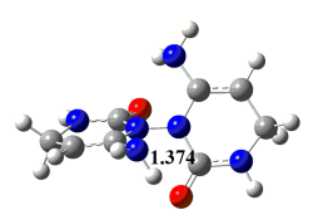

Figure S8. The optimized geometries with indicated bond lengths $(\AA \hat{)})$ for the $\left(\mathbf{C H 6}^{\circ} \mathbf{C H 6}\right)$ Series base pairs(a. closed-shell singlet; b. open-shell broken-symmetry singlet; c. triplet state). 


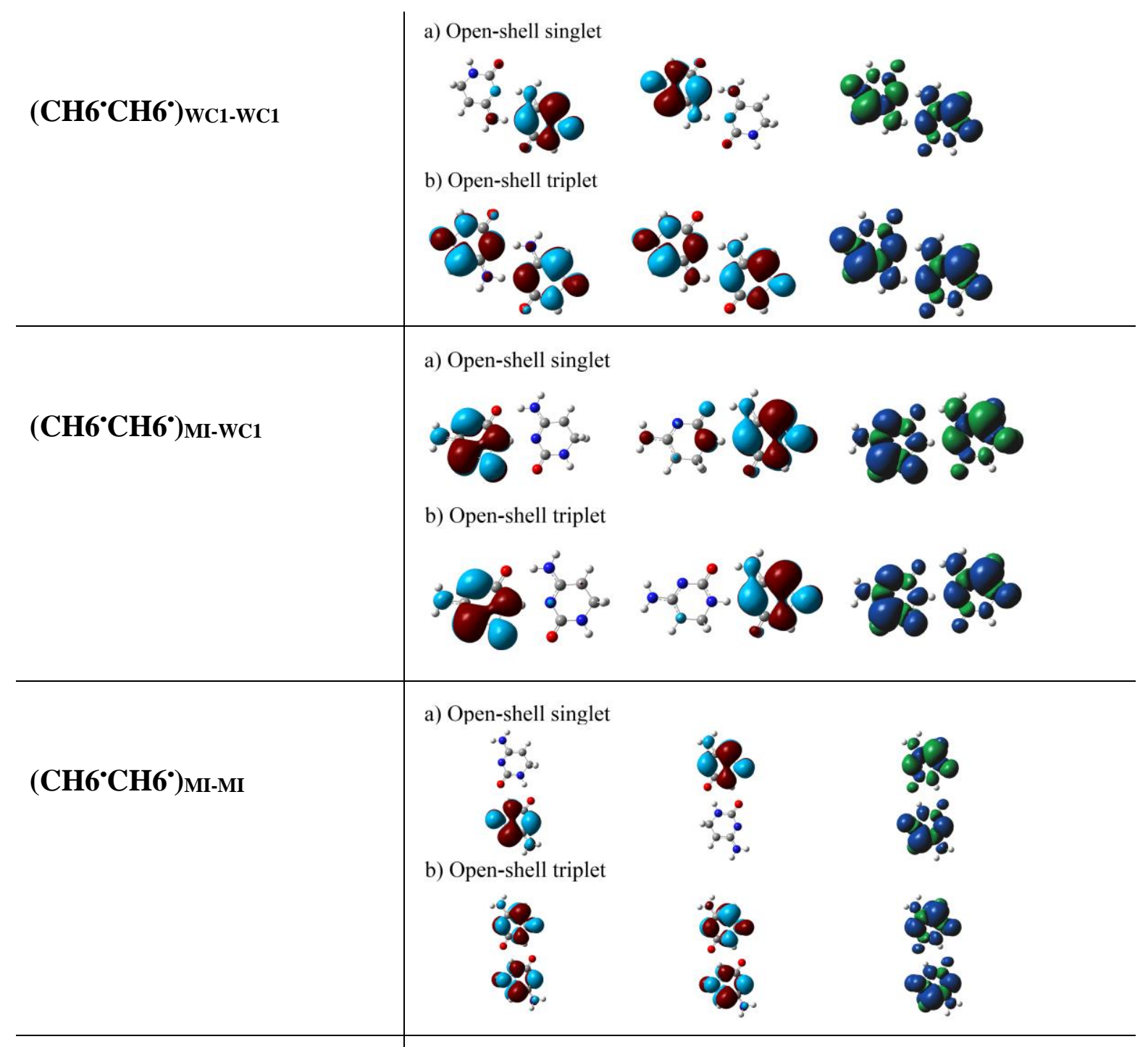

a) Open-shell singlet

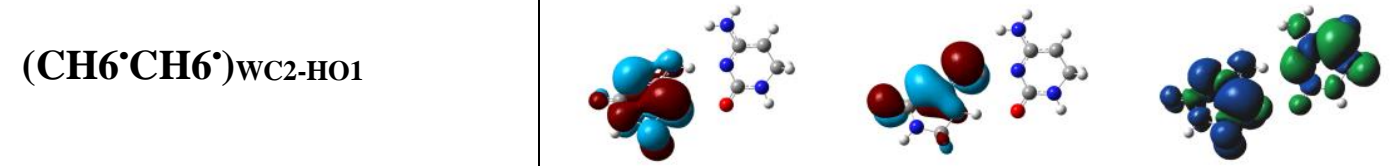

b) Open-shell triplet

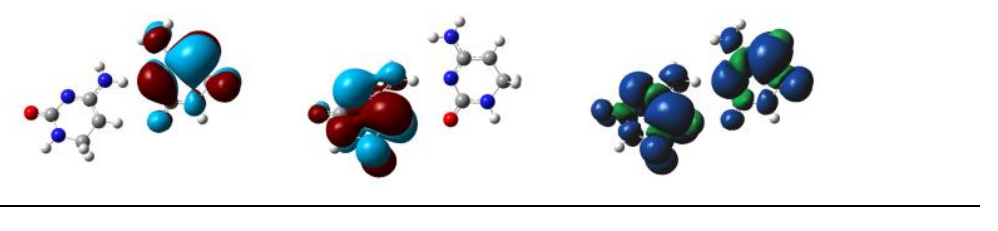

a) Open-shell triplet

\section{$\left(\mathrm{CH6}^{\circ} \mathrm{CH6}^{\circ}\right)_{\mathrm{N} 3-\mathrm{N} 3}$}
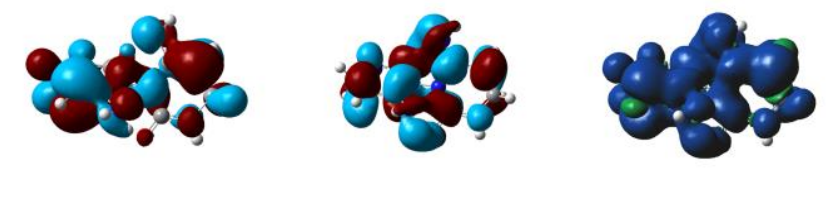

Figure S9. Singly occupied molecular orbitals (SOMOs) and spin density surfaces for open-shell broken-symmetry (BS) singlet and triplet (T) states of the $\left(\mathrm{CH}^{\circ} \mathrm{CH} 6^{\circ}\right) \mathrm{WC1}-\mathrm{WC} 1$, $\left(\mathrm{CH} 6{ }^{\circ} \mathrm{CH} 6{ }^{\circ}\right)_{\mathrm{MI}-\mathrm{WCl}},\left(\mathrm{CH} 6^{\circ} \mathrm{CH} 6^{\circ}\right)_{\mathrm{MI}-\mathrm{MI}},\left(\mathrm{CH}^{\circ} \mathrm{CH} 6^{\circ}\right)_{\mathrm{WC} 2-\mathrm{HO} 1}$ and $\left(\mathrm{CH}^{\circ} \mathrm{CH} 6^{\circ}\right)_{\mathrm{N} 3-\mathrm{N} 3}$ base pair. 
IV) $\left(\mathrm{CH6}^{\circ} \mathrm{CH5}^{\circ}\right)$ series

CS

BS

$\mathbf{T}$

$\left(\mathrm{CH6}^{\circ} \mathrm{CH}^{\circ}\right)^{\circ} \mathrm{wC1}^{\circ} \mathrm{wC1}$
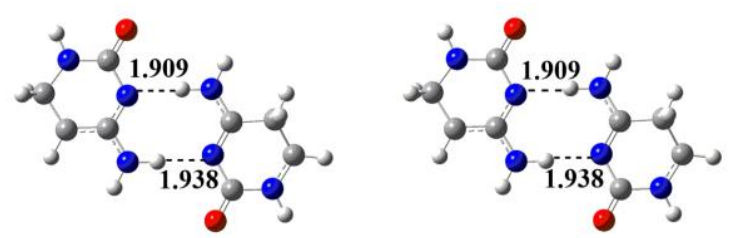

$\left(\mathrm{CH6}^{\circ} \mathrm{CH5}^{\circ}\right)^{\mathrm{MI}-\mathrm{WC1}}$
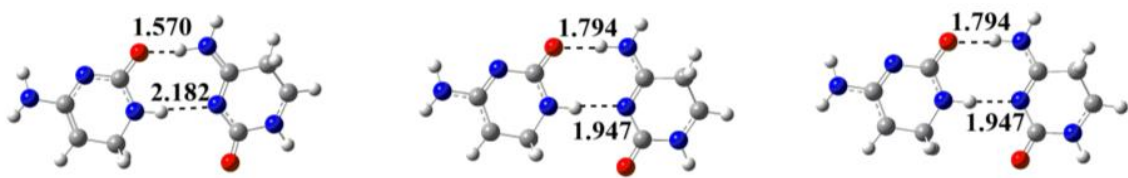

$\left(\mathrm{CH6}^{\circ} \mathrm{CH5}^{\circ}\right)_{\mathrm{MI}-\mathrm{MI}}$
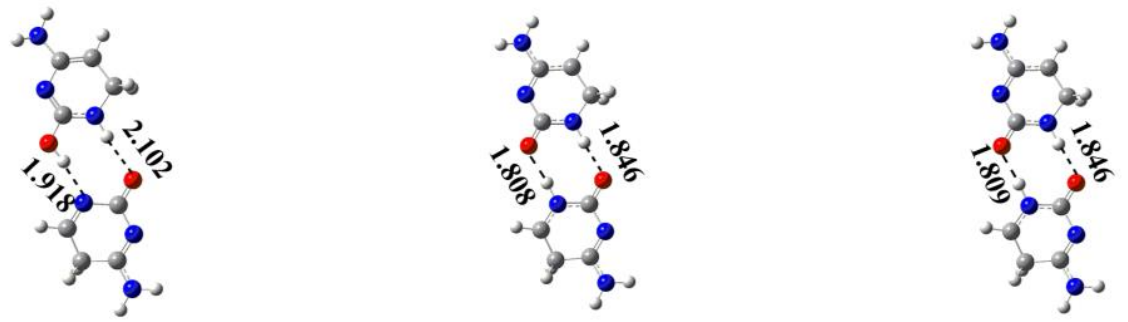

$\left(\mathrm{CH5}^{\circ} \mathrm{CH6}^{\circ}\right)_{\mathrm{MI}-\mathrm{WC1}}$
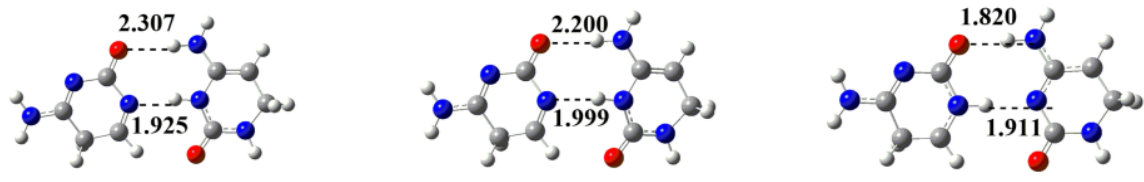

$\left(\mathrm{CH5}^{\circ} \mathrm{CH6}^{\circ}\right) \mathrm{wC2}^{\mathrm{H}} \mathrm{HO}$
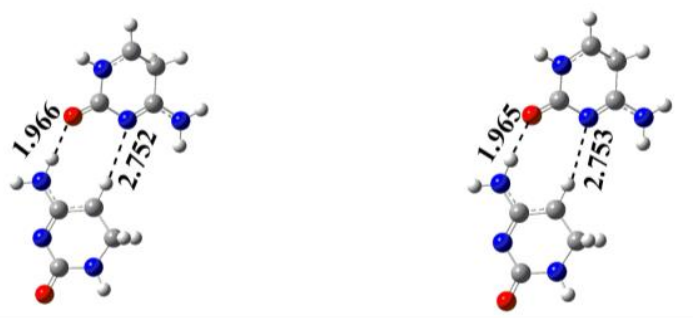

$\left(\mathrm{CH5}^{\circ} \mathrm{CH6}^{\circ}\right)_{\mathrm{N} 3-\mathrm{N} 3}$

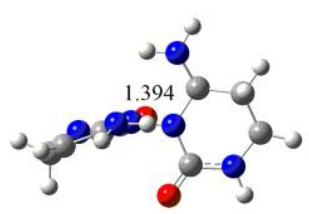

Figure S10. The optimized geometries with indicated bond lengths ( $\AA$ ) for the $\left(\mathrm{CH} 6^{\circ} \mathrm{CH} 5^{\circ}\right)$ Series base pairs(a. closed-shell singlet; b. open-shell broken-symmetry singlet; c. triplet state). 


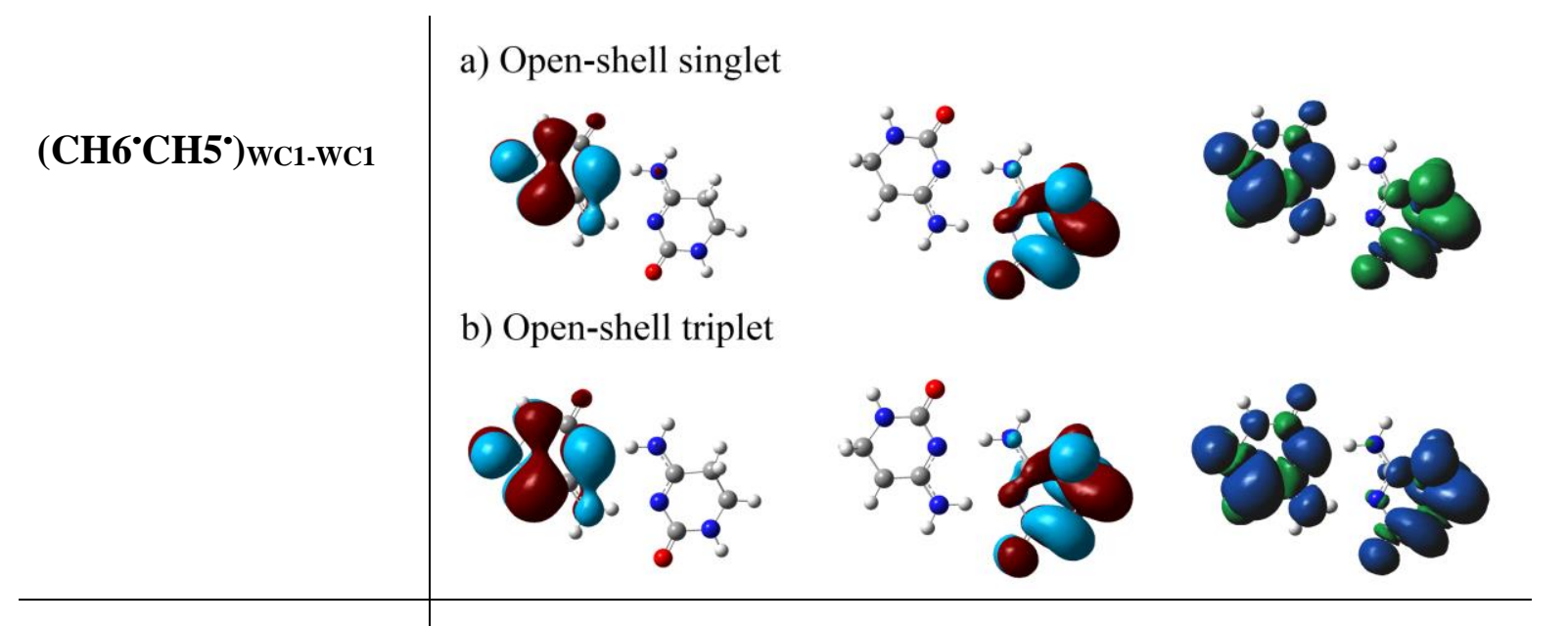

a) Open-shell singlet

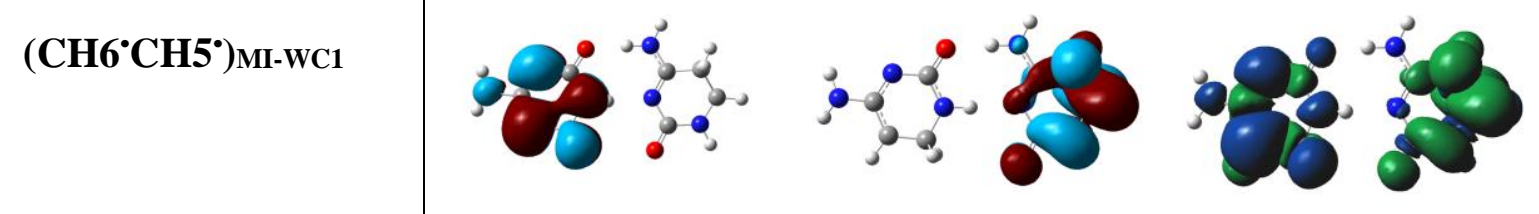

b) Open-shell triplet

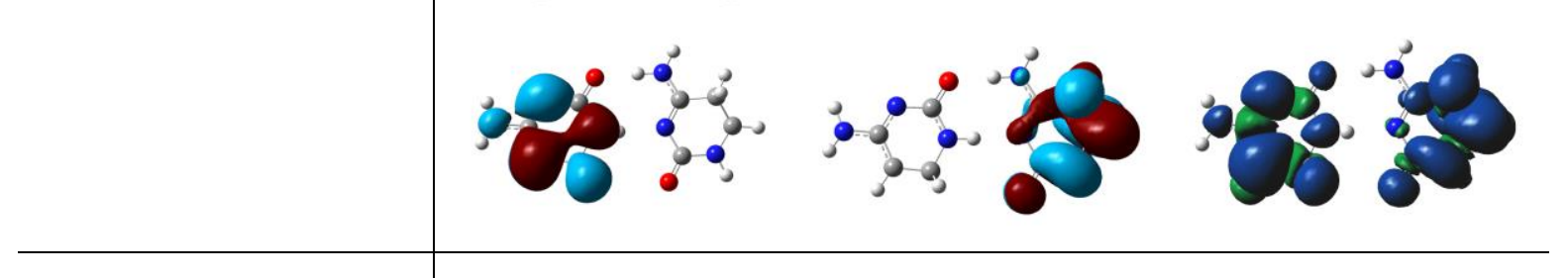

a) Open-shell singlet

\section{(CH6 $\left.^{\circ} \mathrm{CH5}^{\circ}\right)_{\mathrm{MI}-\mathrm{MI}}$}
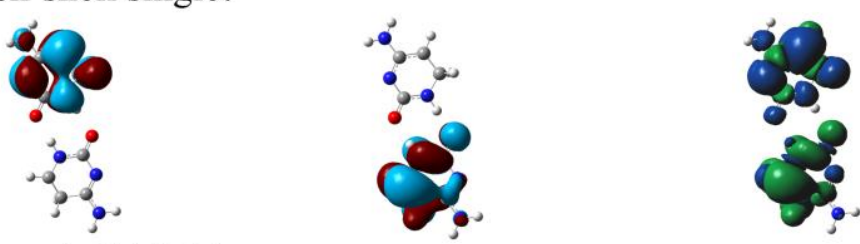

b) Open-shell triplet
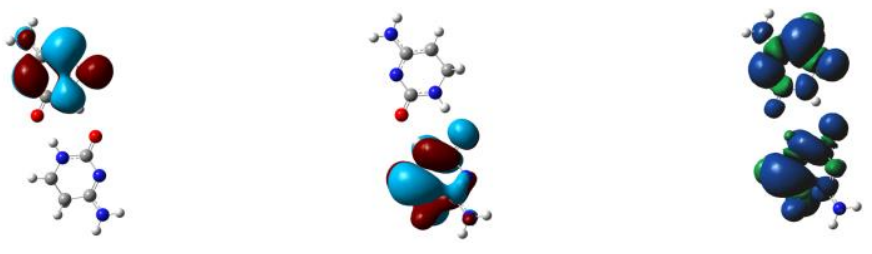

Figure S11. Singly occupied molecular orbitals (SOMOs) and spin density surfaces for open-shell broken-symmetry (BS) singlet and triplet (T) states of the $\left(\mathrm{CH} 6{ }^{\circ} \mathrm{CH} 5^{\circ}\right)$ WC1-WC1, $\left(\mathrm{CH} 6{ }^{\circ} \mathrm{CH} 5^{\circ}\right)_{\mathrm{MI}-\mathrm{WC} 1}$ and $\left(\mathrm{CH} 6^{\circ} \mathrm{CH} 5^{\circ}\right)_{\mathrm{MI}-\mathrm{MI}}$ base pair. 


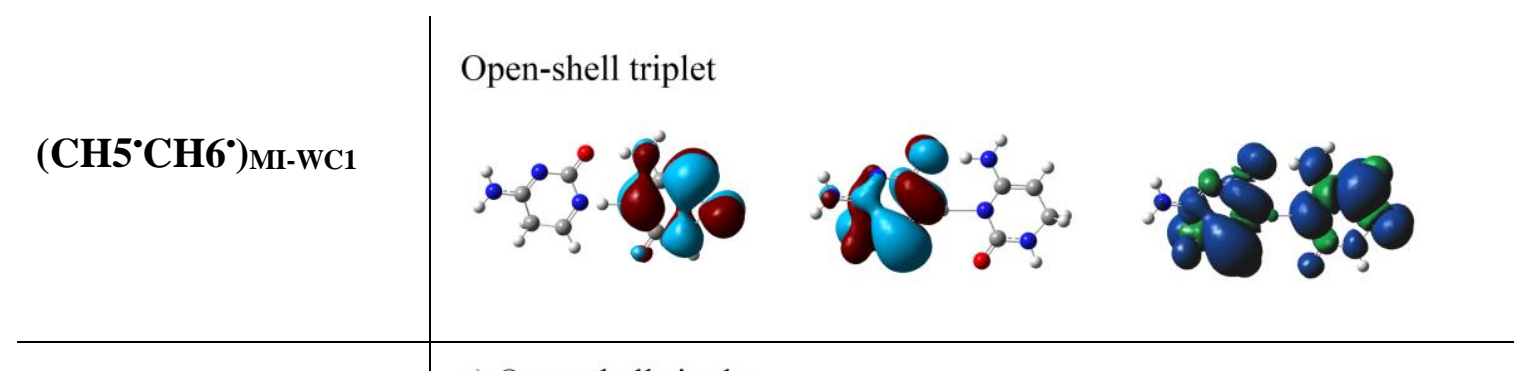

$\left(\mathrm{CH5}^{\circ} \mathrm{CH}^{\circ}\right)_{\mathrm{WC2}-\mathrm{HO}}$

a) Open-shell singlet
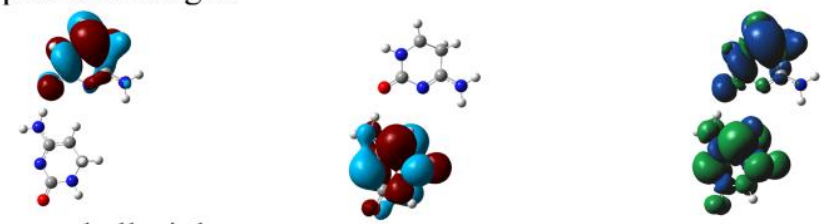

b) Open-shell triplet
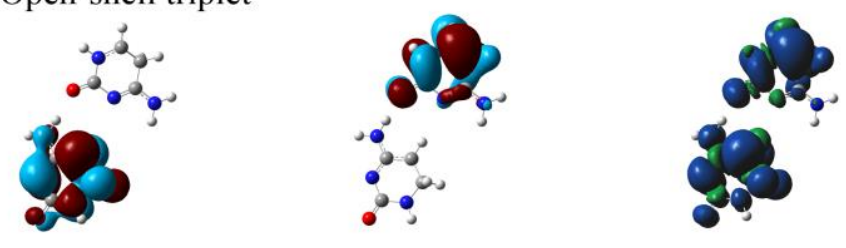

Figure S12. Singly occupied molecular orbitals (SOMOs) and spin density surfaces for open-shell broken-symmetry (BS) singlet and triplet (T) states of the $\left(\mathrm{CH} 5^{\circ} \mathrm{CH} 6^{\circ}\right)_{\mathrm{MI}-\mathrm{WC} 1}$ and $\left(\mathrm{CH} 5^{\circ} \mathrm{CH} 6^{\circ}\right)_{\mathrm{WC} 2-\mathrm{HO} 1}$ base pair.

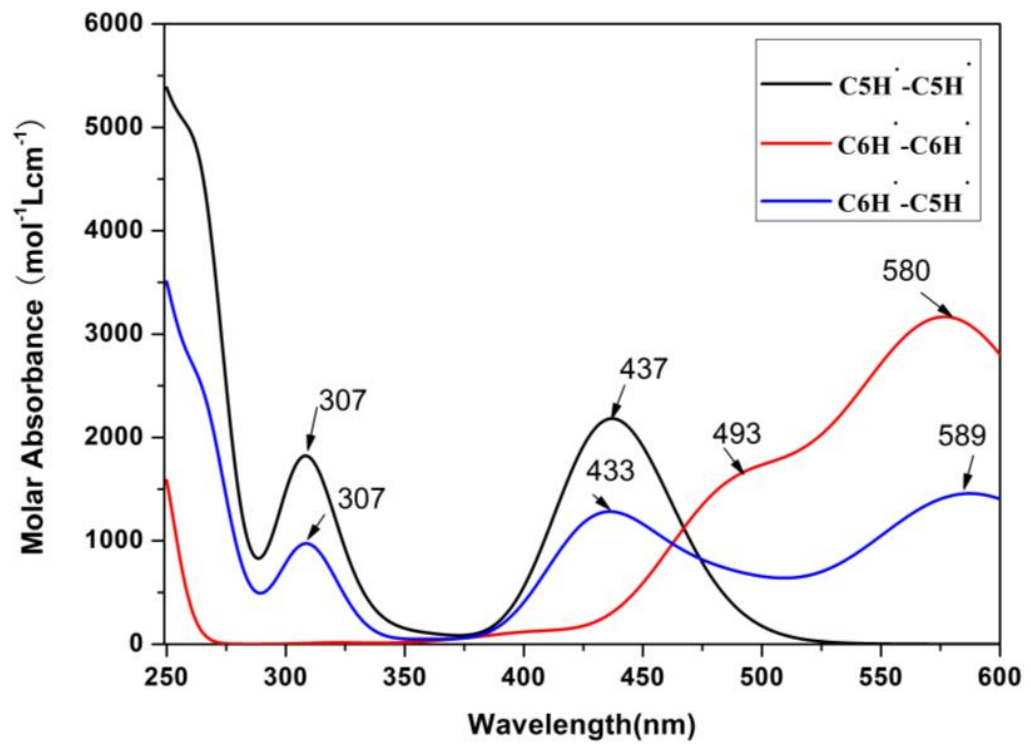

Figure S13. The predicted absorption spectra of $\left(\mathrm{CH} 5^{\circ} \mathrm{CH} 5^{\circ}\right) \mathrm{WC1}-\mathrm{WC} 1,\left(\mathrm{CH} 6^{\circ} \mathrm{CH} 6^{\circ}\right) \mathrm{WC1}-\mathrm{WC} 1$, and $\left(\mathrm{CH} 6^{\circ} \mathrm{CH} 5^{\circ}\right)_{\mathrm{WC} 1-\mathrm{wC}}$. The TDDFT approach was employed to calculate the electronic transition energies of the doubly-reduced species $\left(\mathrm{CH} 5^{\circ} \mathrm{CH} 5^{\circ}\right)$ wC1-wC1, $\left(\mathrm{CH} 6^{\circ} \mathrm{CH} 6^{\circ}\right)$ wC1-wC1 and $\left(\mathrm{CH} 5^{\circ} \mathrm{CH} 6^{\circ}\right)_{\mathrm{WC1}-\mathrm{WC}}$. The absorption visible spectra analysis shows their absorption bands being in the range from $250 \mathrm{~nm}$ to $600 \mathrm{~nm}$. 
V) $\left(\mathrm{CH}^{\circ} \mathrm{CH}^{\circ}\right)$ series

$\left(\mathrm{CH}^{\circ}{ }^{\circ} \mathrm{CH}^{\circ}\right)$ MI-WC2

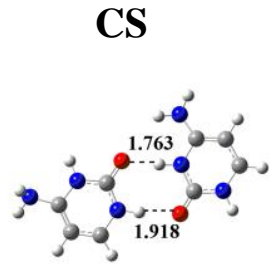

BS

$\mathbf{T}$
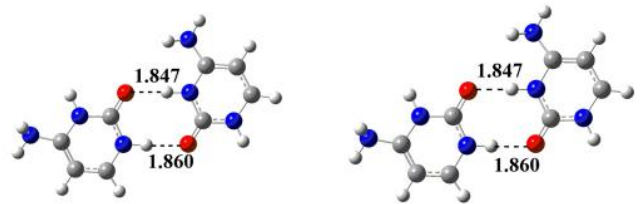

$\left(\mathrm{CH3}^{\circ} \mathrm{CH3}^{\circ}\right)$ MI-MI
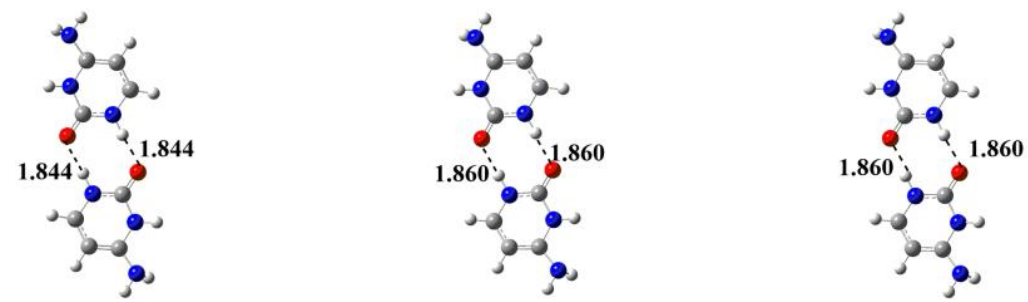

Figure S14. The optimized geometries with indicated bond lengths ( $\AA$ ) for the $\left(\mathrm{CH} 3^{\circ} \mathrm{CH} 3^{\circ}\right)$ Series base pairs (a. closed-shell singlet; b. open-shell broken-symmetry singlet; c. triplet state).

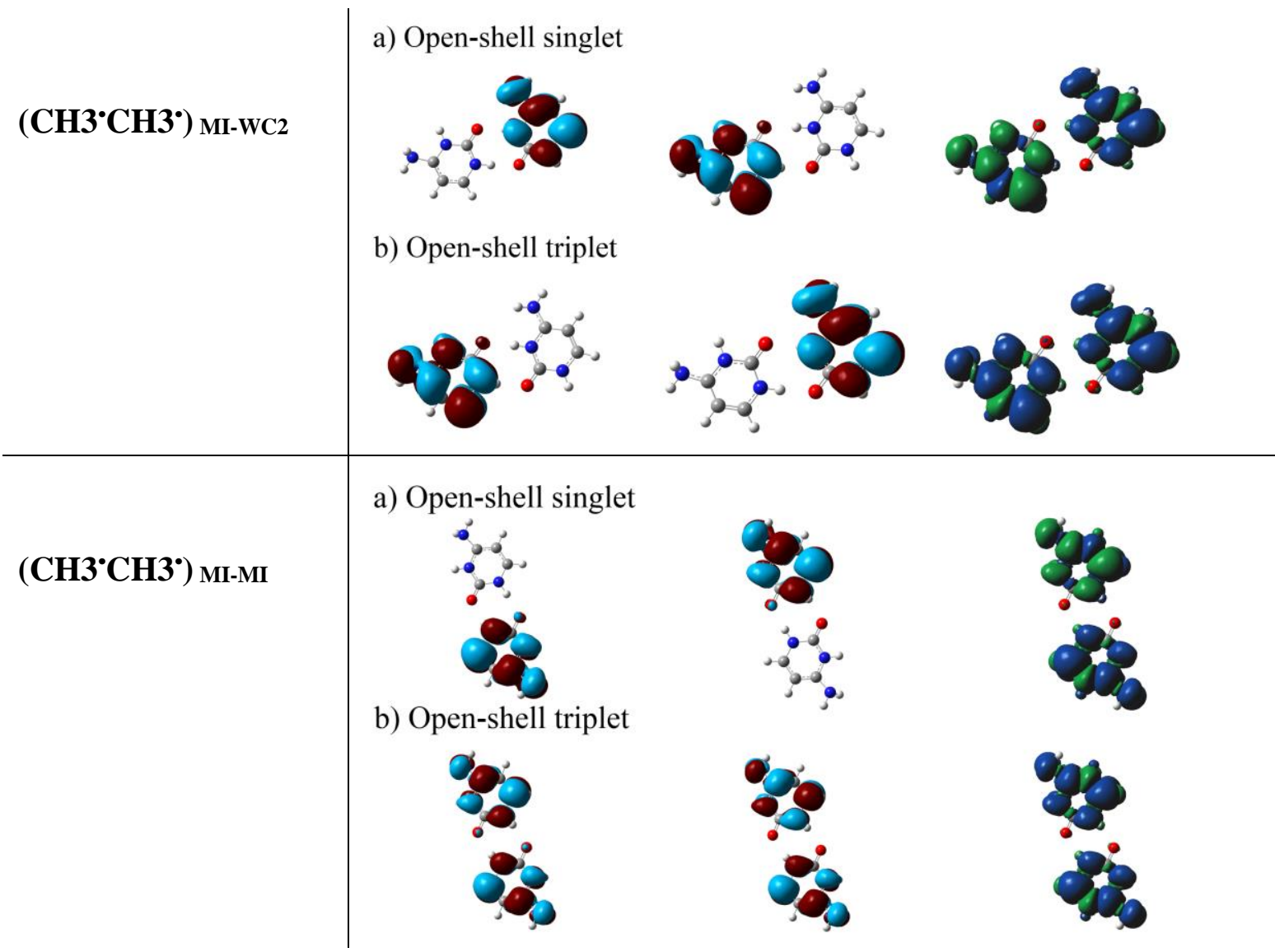

Figure S15. Singly occupied molecular orbitals (SOMOs) and spin density surfaces for open-shell broken-symmetry (BS) singlet and triplet $(\mathrm{T})$ states of the $\left(\mathrm{CH} 3{ }^{\circ} \mathrm{CH} 3^{\circ}\right)_{\mathrm{MI}-\mathrm{WC} 2}$, and $\left(\mathrm{CH} 3{ }^{\circ} \mathrm{CH} 3{ }^{\circ}\right)_{\mathrm{MI}-\mathrm{MI}}$ base pair. 
VI) $\left(\mathrm{CH}^{\circ}{ }^{\circ}{ }^{\circ}\right)$ series

CS

$\left(\right.$ CH5 $\left.^{\circ} \mathrm{C}^{\circ-}\right)$ WC1-WC1

$\left(\mathrm{CH5}^{\circ} \mathrm{C}^{-0}\right)_{\mathrm{MI}-\mathrm{WC1}}$
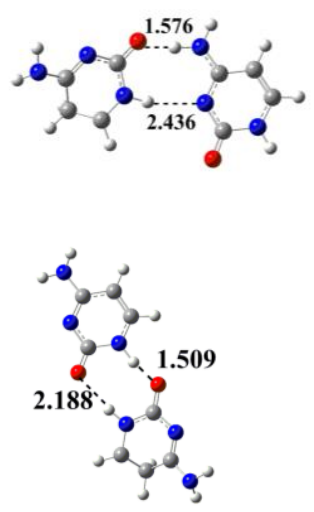

$\left(\mathrm{CH}^{\circ}{ }^{\circ}{ }^{\circ}\right)_{\mathrm{MI}-\mathrm{MI}}$

$\left(\mathrm{CH5}^{\circ} \mathrm{C}^{\circ-}\right)$ WC2-HO1
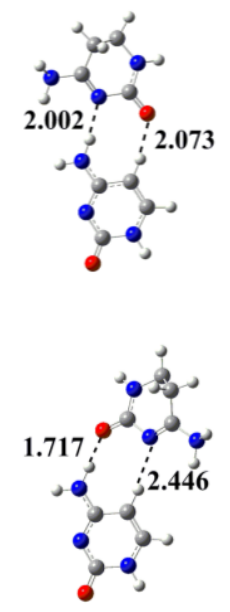

$\left(\mathrm{C}^{\bullet-\mathrm{CH}^{\circ}}\right)_{\mathrm{MI}-\mathrm{WC1}}$
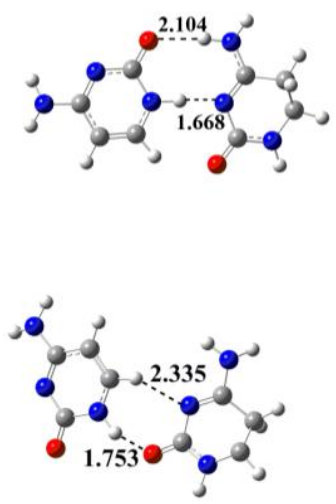

$\mathbf{T}$
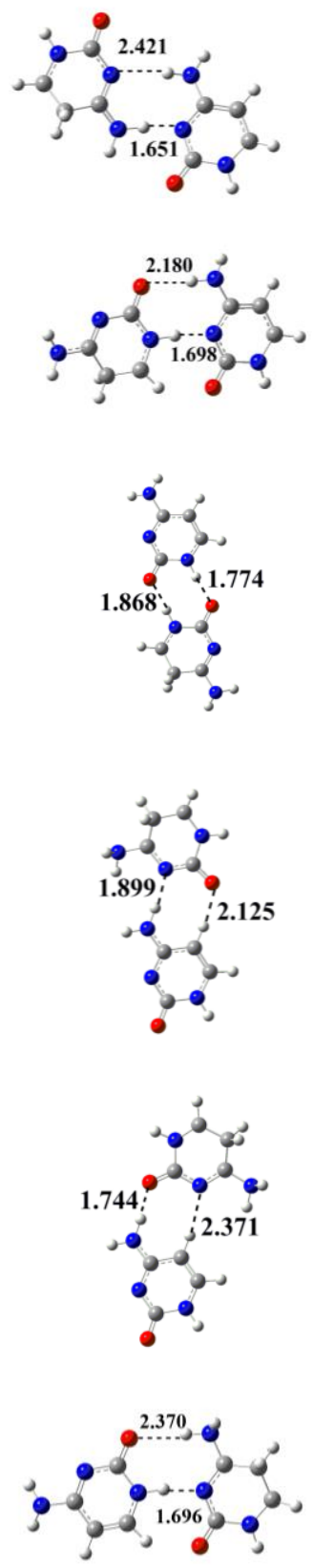

$\left(\mathrm{C}^{\cdot} \mathrm{CH}^{\circ}\right)_{\mathrm{MII}-\mathrm{WC2}}$

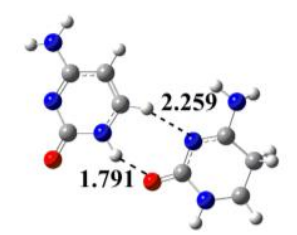

Figure S16. The optimized geometries with indicated bond lengths (Á) for the $\left(\mathrm{CH} 5^{\circ} \mathrm{C}^{*}\right)$ series base pairs(a. closed-shell singlet; b. triplet state). 


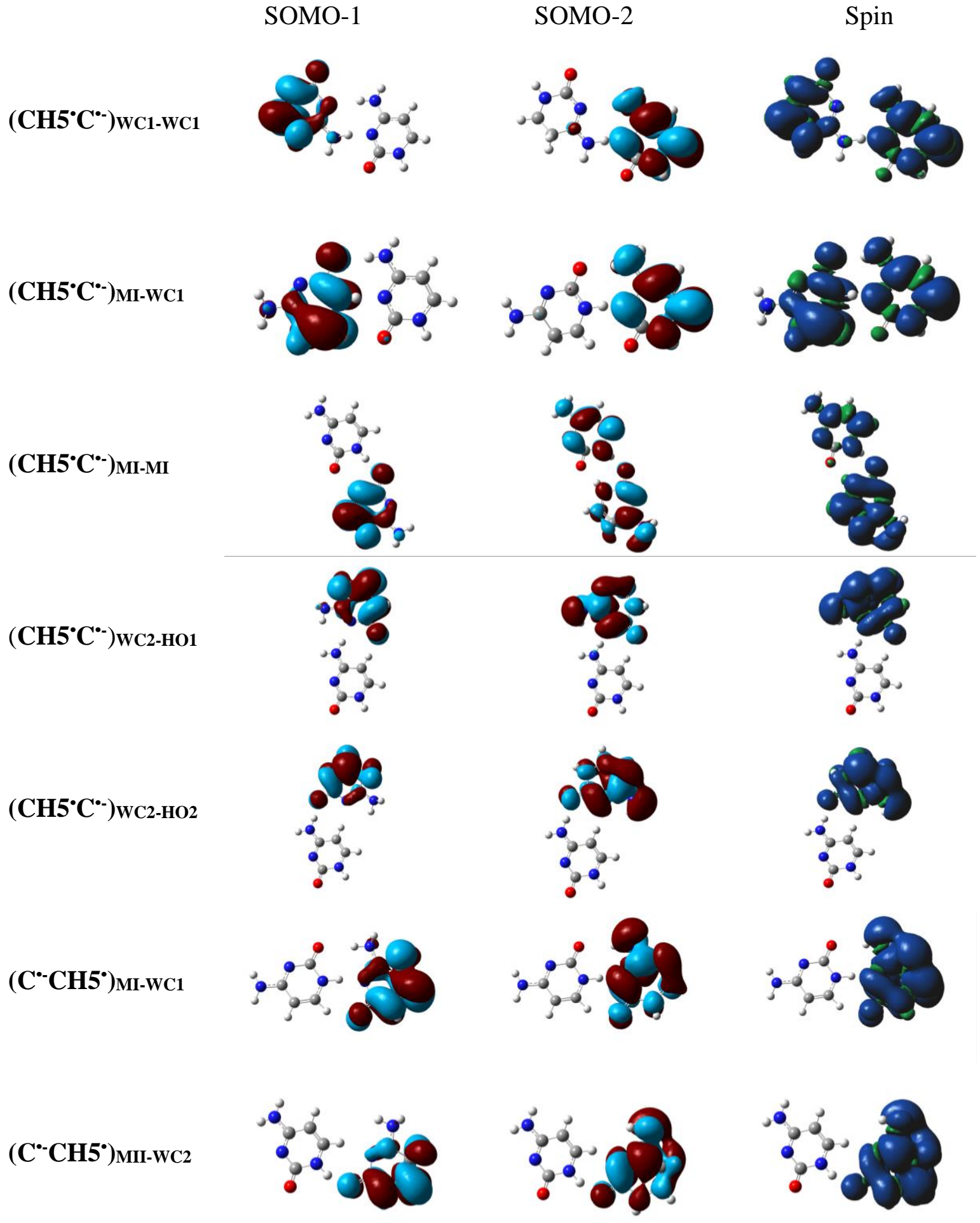

Figure S17. Singly occupied molecular orbitals (SOMOs) and spin density surfaces for triplet (T) states of the $\left(\mathrm{CH} 5^{\circ} \mathrm{C}^{\circ}\right)$ series base pair. 
VII $) \quad\left(\mathrm{CH}^{\circ} \mathrm{C}^{\circ}\right)$ series

$\left(\mathrm{CH6}^{\circ} \mathrm{C}^{\circ-}\right)$ WC1-WC1
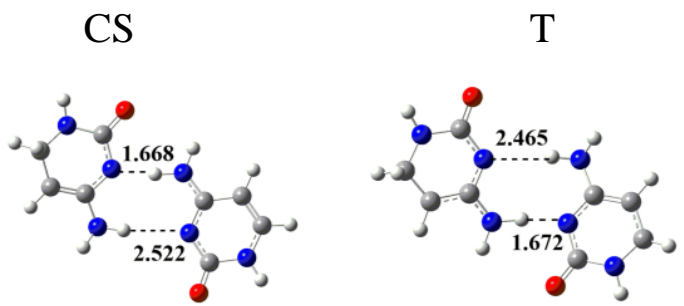

$\left(\mathrm{CH6}^{\circ} \mathrm{C}^{*-}\right)_{\mathrm{MI}-\mathrm{WC1}}$
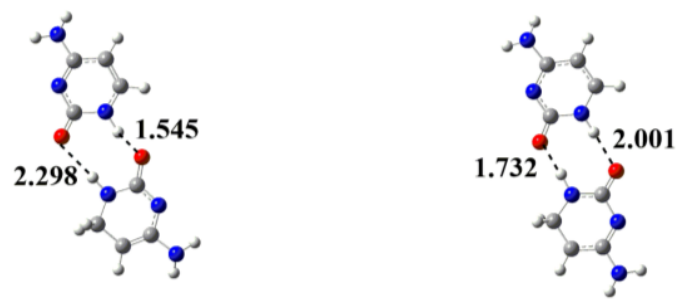

$\left(\mathrm{CH6}^{\circ} \mathrm{C}^{\circ-}\right)_{\mathrm{MI}-\mathrm{MI}}$
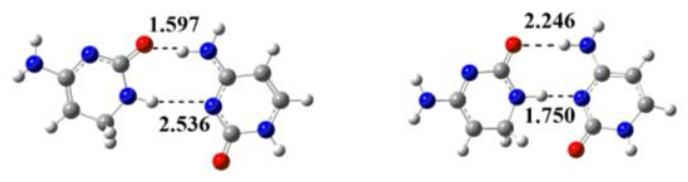

$\left(\mathrm{C}^{\circ-\mathrm{CH}^{\circ}}\right)_{\mathrm{MI}-\mathrm{WC} 1}$
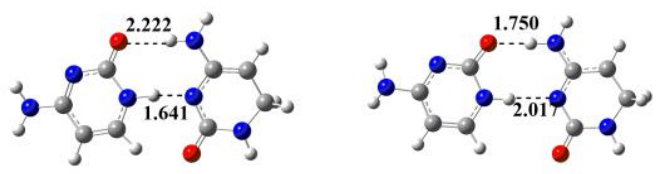

$\left(\mathrm{CH6}^{\circ} \mathrm{C}^{\circ-}\right)$ WC2-HO1
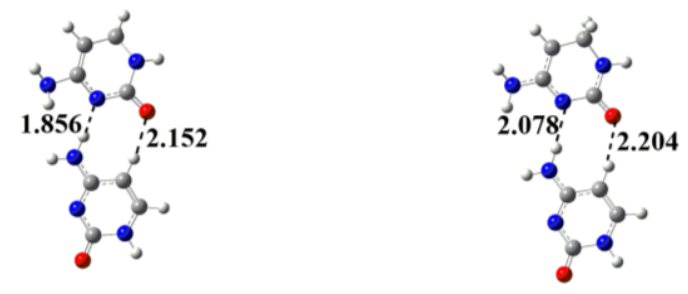

$\left(\right.$ CH6 $\left.^{\circ}{ }^{\circ-}\right)$ WC1-Ho
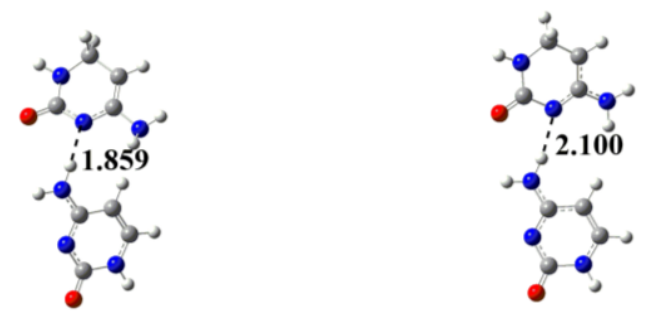

$\left(\right.$ CH6 $\left.^{\circ} \mathrm{C}^{\circ-}\right)$ WC2-HO
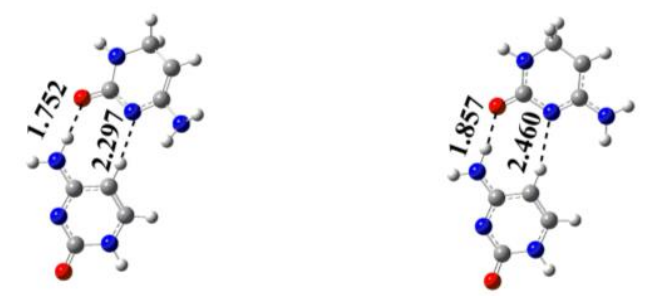

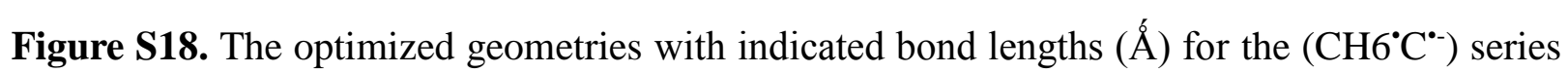
base pairs(a. closed-shell singlet; b. triplet state). 


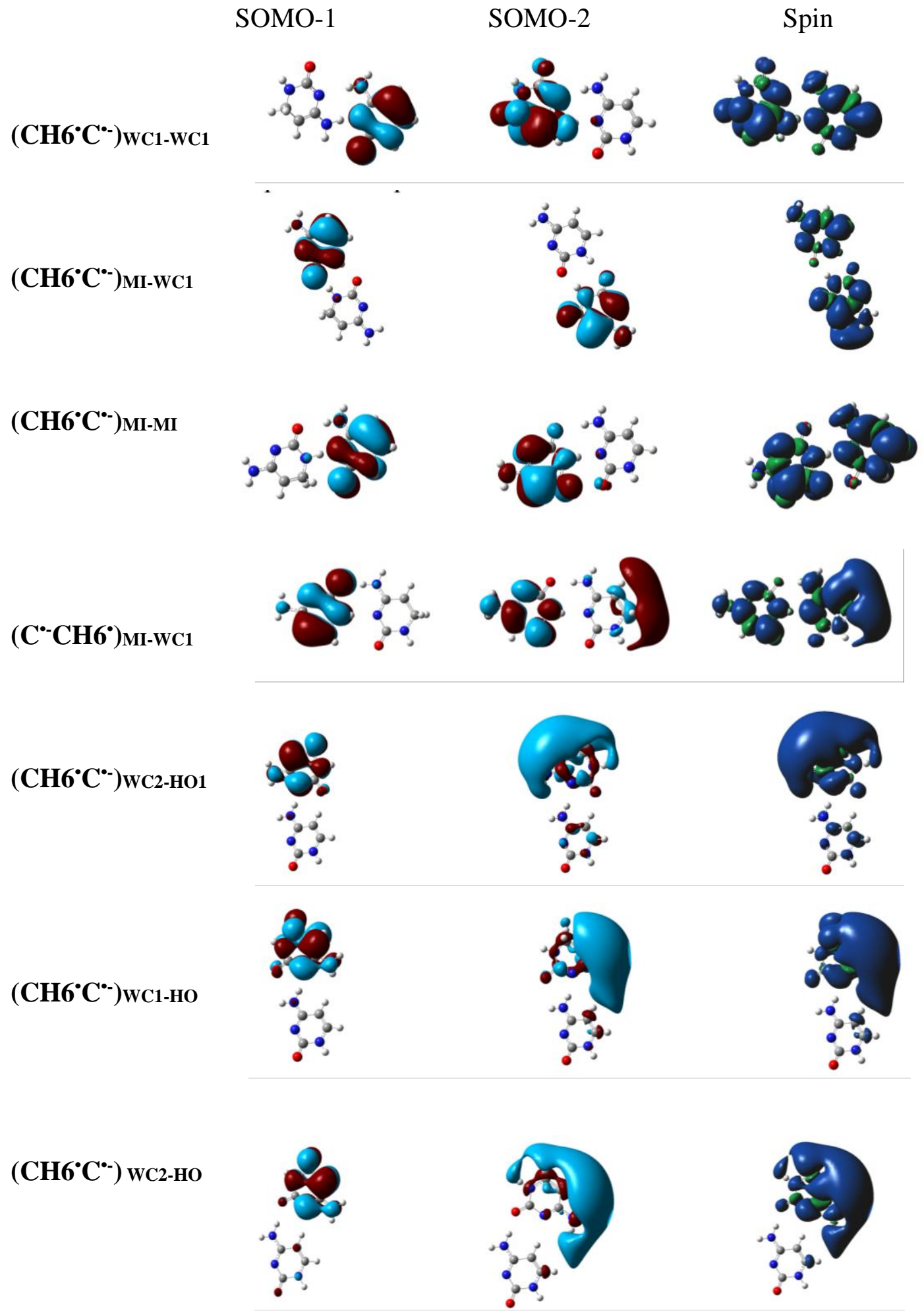

Figure S19. Singly occupied molecular orbitals (SOMOs) and spin density surfaces for triplet (T) states of the $\left(\mathrm{CH}^{\circ} \mathrm{C}^{\circ-}\right)$ series base pair. 


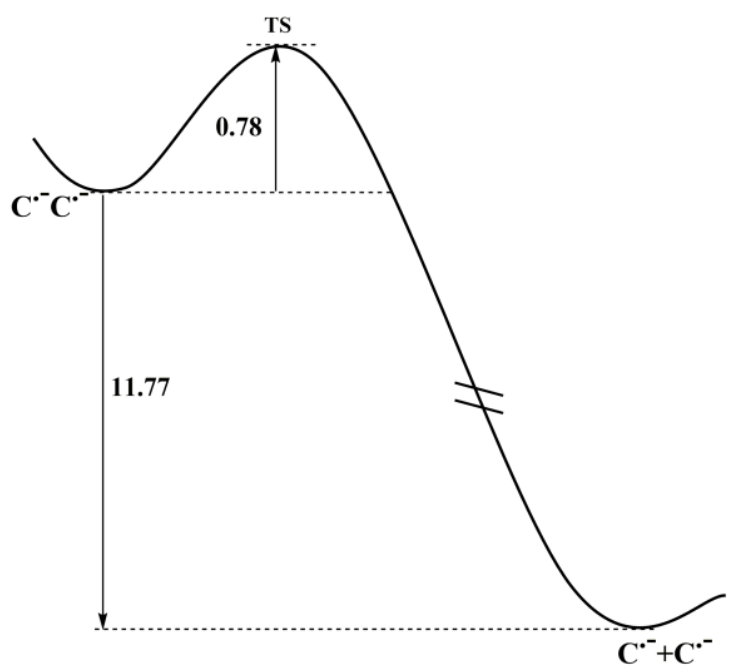

Figure 20. The potential energy curves and energies $(\mathrm{kcal} / \mathrm{mol})$ along the $\left(\mathrm{C}^{\bullet-}\right) \mathrm{N}-\mathrm{H} \ldots \mathrm{N}\left(\mathrm{C}^{\bullet-}\right)$

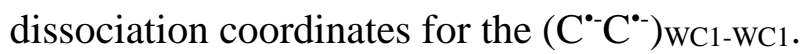

\section{$\left(\mathrm{CH6}^{\circ} \mathrm{CH6}^{\circ}\right)_{\pi \pi}$ Series}

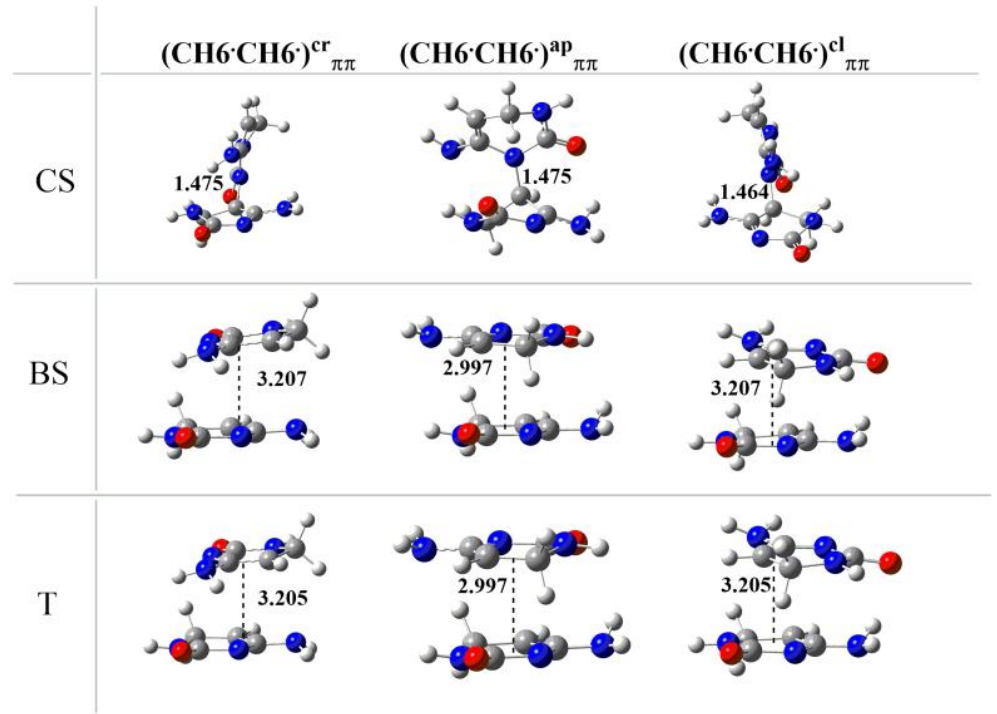

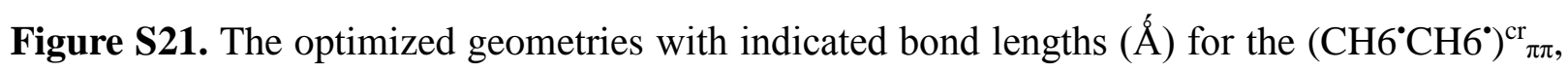
$\left(\mathrm{CH}^{\circ} \mathrm{CH} 6^{\circ}\right)^{\text {ap }}{ }_{\pi \pi}$ and $\left(\mathrm{CH}^{\circ} \mathrm{CH}^{\circ}\right)^{\mathrm{cl}}{ }_{\pi \pi}$ base pairs. $\mathrm{CS}$, BS and $\mathrm{T}$ denote closed-shell singlet, open-shell broken-symmetry singlet, and triplet state, respectively. For the $\left(\mathrm{CH} 6^{\circ} \mathrm{CH} 6^{\circ}\right)_{\pi}$ base pairs, the geometries of closed-shell singlet state have the $\mathrm{N}_{3}\left(\mathrm{CH} 6^{\circ}\right)-\mathrm{C}_{5}\left(\mathrm{CH} 6^{\circ}\right)$ bond between the two $\mathrm{CH}^{\circ}$ bases. 


\section{$\left(\mathrm{CH5}^{\circ}{ }^{\circ} 6^{\circ}\right)_{\pi \pi}$ Series}

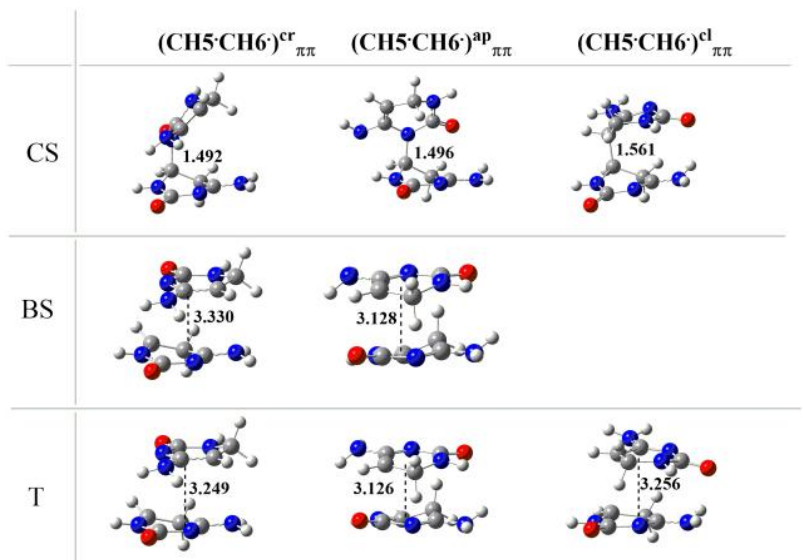

Figure S22. The optimized geometries with indicated bond lengths $(\AA \hat{)})$ for $\left(\mathrm{CH} 5^{\circ} \mathrm{CH} 6^{\circ}\right)^{\mathrm{cr}} \pi \pi$, $\left(\mathrm{CH} 5^{\circ} \mathrm{CH} 6^{\circ}\right)^{\mathrm{ap}}{ }_{\pi \pi}$ and $\left(\mathrm{CH}^{\circ} \mathrm{CH} 6^{\circ}\right)^{\mathrm{cl}}{ }_{\pi \pi}$ base pairs. $\mathrm{CS}$, BS and $\mathrm{T}$ denote closed-shell singlet, open-shell BS singlet, and triplet state, respectively. In the closed-shell singlet state for $\left(\mathrm{CH} 5^{\circ} \mathrm{CH} 6^{\circ}\right)^{\mathrm{cr}}{ }_{\pi \pi},\left(\mathrm{CH} 5^{\circ} \mathrm{CH} 6^{\circ}\right)^{\mathrm{ap}}{ }_{\pi \pi}$ and $\left(\mathrm{CH} 5^{\circ} \mathrm{CH} 6^{\circ}\right)^{\mathrm{cl}}{ }_{\pi \pi}$, the $\mathrm{N}_{3}-\mathrm{C}_{6}, \mathrm{~N}_{3}-\mathrm{C}_{6}$ and $\mathrm{C}_{5}-\mathrm{C}_{6}$ cross link between $\mathrm{CH}^{\circ}$ and $\mathrm{CH}^{\circ}$, respectively. When $\left(\mathrm{CH}^{\circ} \mathrm{CH} 6^{\circ}\right)^{\mathrm{cl}}{ }_{\pi \pi}$ conformations in BS state, the obtained structure $\mathrm{N}_{4}-\mathrm{C}_{6}$ cross links between $\mathrm{N}_{4}$ of $\mathrm{CH}^{\circ}$ and $\mathrm{C}_{6}$ of the other $\mathrm{CH} 5^{\circ}$, thus $\left(\mathrm{CH} 5^{\circ} \mathrm{CH} 6^{\circ}\right)^{\mathrm{cl}} \pi \pi$ only have $\mathrm{CS}$ and T states.

\section{$\left(\mathrm{CH3}^{\circ} \mathrm{CH3}^{\circ}\right)_{\pi \pi}$ Series}

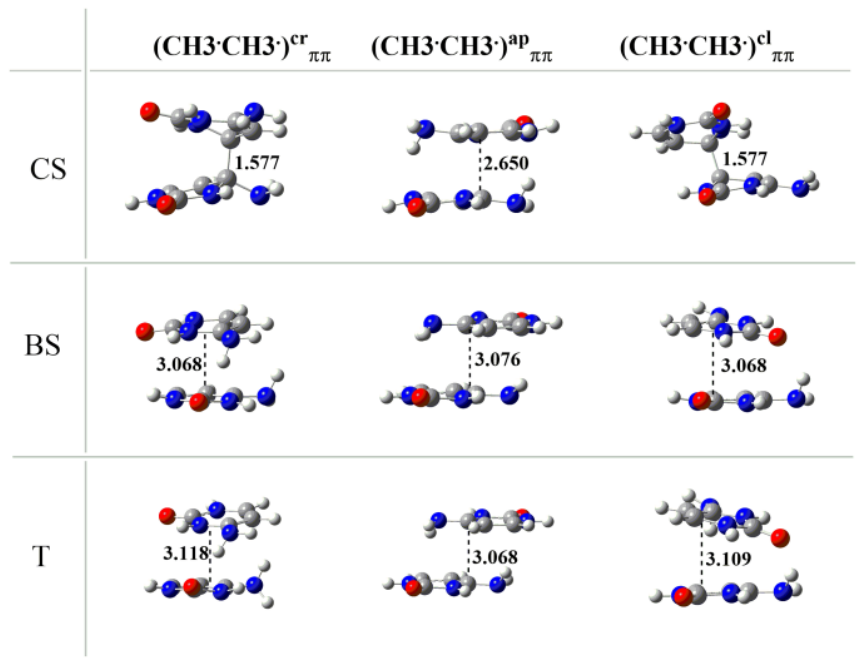

Figure S23. The optimized geometries with indicated bond lengths $(\AA \hat{)})$ for $\left(\mathrm{CH} 3^{\circ} \mathrm{CH} 3^{\circ}\right)^{\mathrm{cr}}{ }_{\pi}$, $\left(\mathrm{CH} 3^{\circ} \mathrm{CH} 3^{\circ}\right)^{\text {ap }}{ }_{\pi \pi}$ and $\left(\mathrm{CH}^{\circ} \mathrm{CH} 3^{\circ}\right)^{\mathrm{cl}}{ }_{\pi \pi}$ base pairs. $\mathrm{CS}$, BS and $\mathrm{T}$ denote closed-shell singlet, open-shell BS singlet, and triplet state, respectively. In the closed-shell singlet state for $\left(\mathrm{CH} 3^{\circ} \mathrm{CH} 3^{\circ}\right)^{\mathrm{cr}}{ }_{\pi \pi}$, and $\left(\mathrm{CH}^{\circ}{ }^{\circ} \mathrm{CH} 3^{\circ}\right)^{\mathrm{cl}}{ }_{\pi \pi}$, the $\mathrm{C}_{6}-\mathrm{C}_{4}$ and $\mathrm{C}_{4}-\mathrm{C}_{6}$ cross link between $\mathrm{CH}^{\circ}$ and $\mathrm{CH} 3^{\circ}$, respectively. 


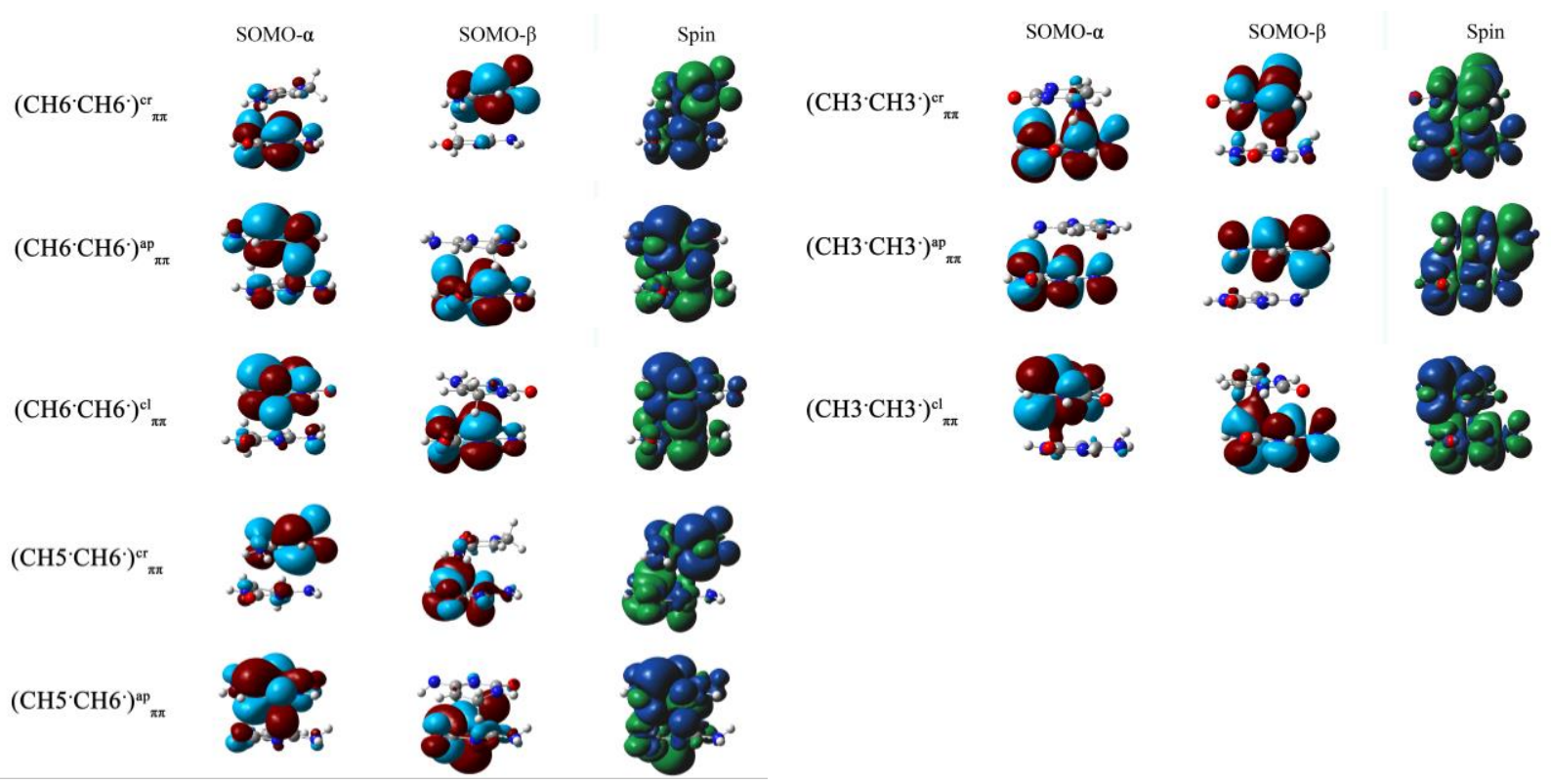

Figure S24. Singly occupied molecular orbitals (SOMOs) and spin density surfaces for open-shell broken-symmetry (BS) singlet states of the $\left(\mathrm{CH} 6{ }^{\circ} \mathrm{CH} 6^{\circ}\right)_{\pi \pi},\left(\mathrm{CH}^{\circ} \mathrm{CH} 6^{\circ}\right)_{\pi \pi}$, $\left(\mathrm{CH} 5^{\circ} \mathrm{CH} 6^{\circ}\right)_{\pi \pi}$ and $\left(\mathrm{CH} 3^{\circ} \mathrm{CH} 3^{\circ}\right)_{\pi \pi}$ base pair.

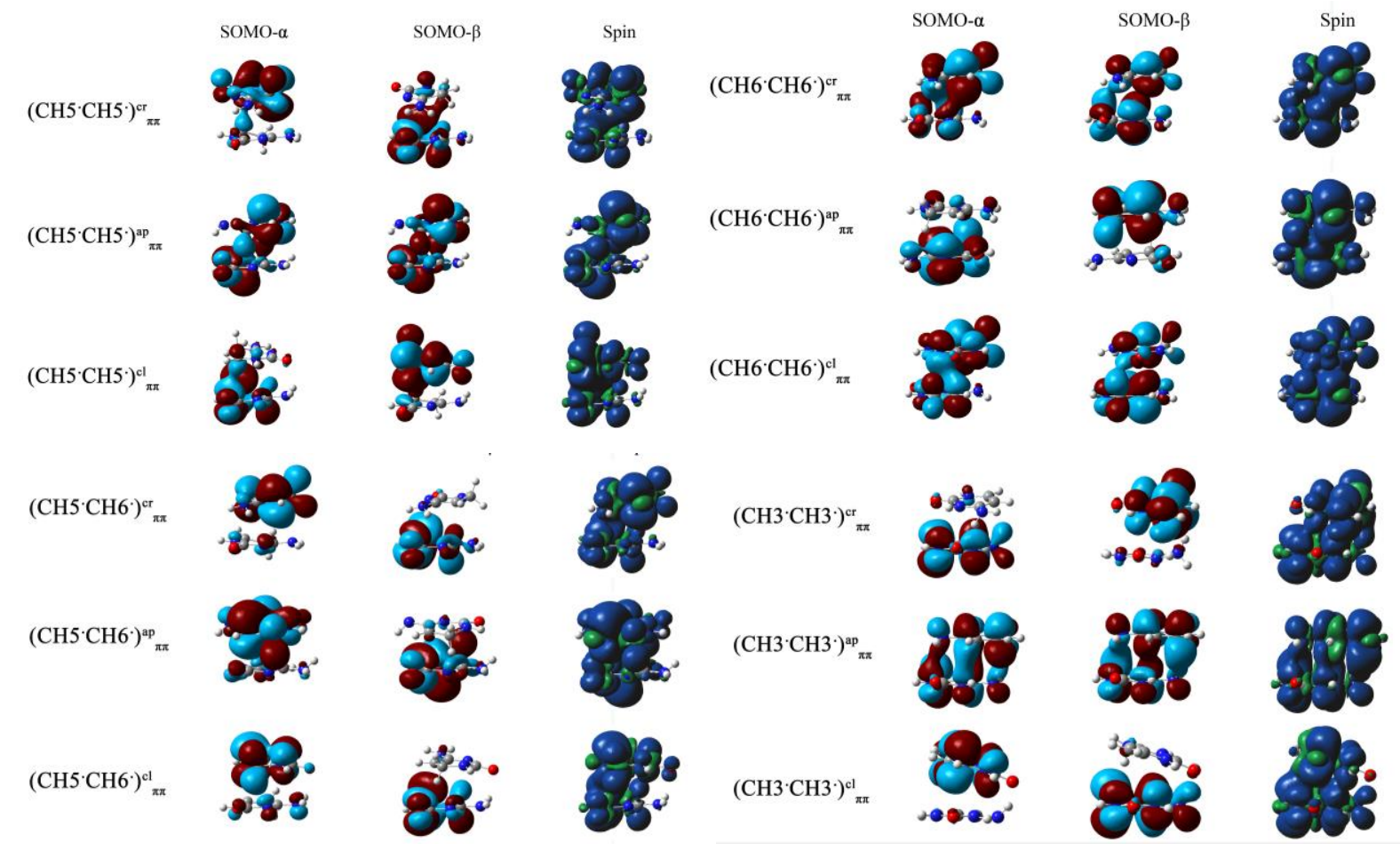

Figure S25. Singly occupied molecular orbitals (SOMOs) and spin density surfaces for triplet (T) states of the $\left(\mathrm{CH} 5^{\circ} \mathrm{CH} 5^{\circ}\right)_{\pi \pi},\left(\mathrm{CH} 6^{\circ} \mathrm{CH} 6^{\circ}\right)_{\pi \pi},\left(\mathrm{CH} 5^{\circ} \mathrm{CH} 6^{\circ}\right)_{\pi \pi}$ and $\left(\mathrm{CH} 3^{\circ} \mathrm{CH} 3^{\circ}\right)_{\pi \pi}$ base pair. 